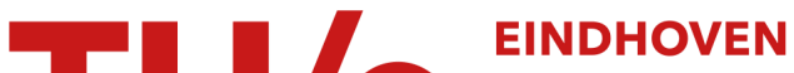 \\ UNIVERSITY OF \\ TECHNOLOGY
}

\section{Eigenmode analysis of scalar transport in distributive mixing}

\section{Citation for published version (APA):}

Singh, M. K., Speetjens, M. F. M., \& Anderson, P. D. (2009). Eigenmode analysis of scalar transport in distributive mixing. Physics of Fluids, 21(9), 093601-1/17. [093601]. https://doi.org/10.1063/1.3231601

DOI:

10.1063/1.3231601

Document status and date:

Published: 01/01/2009

\section{Document Version:}

Publisher's PDF, also known as Version of Record (includes final page, issue and volume numbers)

\section{Please check the document version of this publication:}

- A submitted manuscript is the version of the article upon submission and before peer-review. There can be important differences between the submitted version and the official published version of record. People interested in the research are advised to contact the author for the final version of the publication, or visit the $\mathrm{DOI}$ to the publisher's website.

- The final author version and the galley proof are versions of the publication after peer review.

- The final published version features the final layout of the paper including the volume, issue and page numbers.

Link to publication

\section{General rights}

Copyright and moral rights for the publications made accessible in the public portal are retained by the authors and/or other copyright owners and it is a condition of accessing publications that users recognise and abide by the legal requirements associated with these rights.

- Users may download and print one copy of any publication from the public portal for the purpose of private study or research.

- You may not further distribute the material or use it for any profit-making activity or commercial gain

- You may freely distribute the URL identifying the publication in the public portal.

If the publication is distributed under the terms of Article 25fa of the Dutch Copyright Act, indicated by the "Taverne" license above, please follow below link for the End User Agreement:

www.tue.nl/taverne

Take down policy

If you believe that this document breaches copyright please contact us at:

openaccess@tue.nl

providing details and we will investigate your claim. 


\title{
Eigenmode analysis of scalar transport in distributive mixing
}

\author{
Mrityunjay K. Singh, Michel F. M. Speetjens, and Patrick D. Anderson ${ }^{\text {a) }}$ \\ Department of Mechanical Engineering, Eindhoven University of Technology, P.O. Box 513, \\ 5600 MB Eindhoven, The Netherlands
}

(Received 3 June 2009; accepted 13 July 2009; published online 22 September 2009)

\begin{abstract}
In this study, we explore the spectral properties of the distribution matrices of the mapping method and its relation to the distributive mixing of passive scalars. The spectral (or eigenvector-eigenvalue) decomposition of these matrices constitutes discrete approximations to the eigenmodes of the continuous advection operator in periodic flows. The eigenvalue spectrum always lies within the unit circle and due to mass conservation, always accommodates an eigenvalue equal to one with trivial (uniform) eigenvector. The asymptotic state of a fully chaotic mixing flow is dominated by the eigenmode corresponding with the eigenvalue closest to the unit circle ("dominant eigenmode"). This eigenvalue determines the decay rate; its eigenvector determines the asymptotic mixing pattern. The closer this eigenvalue value is to the origin, the faster is the homogenization by the chaotic mixing. Hence, its magnitude can be used as a quantitative mixing measure for comparison of different mixing protocols. In nonchaotic cases, the presence of islands results in eigenvalues on the unit circle and associated eigenvectors demarcating the location of these islands. Eigenvalues on the unit circle thus are qualitative indicators of inefficient mixing; the properties of its eigenvectors enable isolation of the nonmixing zones. Thus important fundamental aspects of mixing processes can be inferred from the eigenmode analysis of the mapping matrix. This is elaborated in the present paper and demonstrated by way of two different prototypical mixing flows: the time-periodic sine flow and the spatially periodic partitioned-pipe mixer. (c) 2009 American Institute of Physics.
\end{abstract} [doi:10.1063/1.3231601]

\section{INTRODUCTION}

Numerous studies have been reported in literature regarding the eigenmode (or "spectral") analysis of transport of passive scalars in time-periodic flows. ${ }^{1-10}$ In general, the evolution of a scalar field is governed by linear advectiondiffusion equations, which allows an analysis in terms of eigenmodes. Such eigenmode analyses reveal the exponential decay of the scalar field, perhaps modulated by oscillations if the eigenvalues are complex. ${ }^{4}$ Pierrehumbert ${ }^{1}$ first observed that in a diffusive tracer field stirred by a timeperiodic velocity field, persistent concentration patterns formed within just a few stirring periods with exponentially decaying intensity as the tracer field approached the fully mixed state. This exponentially modulated time-periodic behavior and the complex spatial structure prompted Pierrehumbert to call the repeating patterns strange eigenmodes. Strange eigenmodes have also been observed experimentally ${ }^{11,12}$ as well as in aperiodic flows, in both numerical and experimental studies. ${ }^{1,11}$ In the limit of arbitrarily small diffusivity, the concentration field is not smooth anymore and sharp gradients in concentration field develop due to the emergence of strange eigenmodes. The dominant one among these eigenmodes is called the strange eigenmode, although there may be several of comparable importance. The abovementioned studies investigated the advective-diffusive transport in terms of eigenmode decompositions of the advectiondiffusion operator, thereby focusing on the evolution from an

a)Electronic mail: p.d.anderson@tue.nl. URL: http://www.mate.tue.nl. initial inhomogeneous state toward a final homogeneous state. The most relevant properties in this homogenization process are first, the decay rate of the dominant eigenmode and second, its spatial structure.

The present study concentrates on the purely advective limit of scalar transport (i.e., distributive mixing) and performs the eigenmode analysis of the advection operator of time-periodic and spatially periodic flows in terms of the spectral properties of the mapping matrix obtained via the mapping method. The mapping method, originally suggested by Spencer and Wiley, ${ }^{13}$ describes the transport of a conservative quantity, like concentration, from one state to another by means of a mapping matrix. The elements of the matrix contain the discretized fractions of fluid transported from an initial cross section to a final one (for spatially periodic flows) or from an initial time to a final time (for timeperiodic flows), i.e., the mapping method concerns purely advective transport. In a range of studies the mapping matrix has been very successfully applied to optimize prototypical and industrial mixing flows. ${ }^{14-17}$ The elegance of the method is that a set of matrices is only created once, and then multiplied to a vector describing the concentration distribution in the domain. This way a large number of mixing protocols can be fairly easy evaluated and compared by changing the order of the matrices. The original idea of Spencer and Wiley ${ }^{13}$ was to study the properties of the matrix or the denseness of powers of the matrix, as it should contain all spatial complexities, but this was not feasible on grounds of limited computing facilities. Anderson et al. ${ }^{18}$ first studied the properties of the eigenvalues of the mapping matrix and 
their corresponding eigenvectors in relation to the mixing properties of chaotic flows. In this paper, the spectral decomposition of the mapping matrix is employed to specifically identify inhomogeneities in mixing patterns (i.e., elliptic islands). This eigenmode analysis enables the efficient prediction of possible inhomogeneous states - and thus of possibly poor mixing - and several advantages over "classical" tools as, e.g., Poincaré sectioning, are discussed. It furthermore enables a quantitative mixing analysis via the estimation of characteristic times for homogeneous states to set in and the size of nonmixing zones.

The paper is organized as follows. Section II provides a concise review of eigenmode decompositions employed for the analysis of advection (diffusion) problems. The mapping method for distributive mixing is introduced in Sec. III. Section IV reconciles the mapping method with the eigenmode decompositions and ties the mixing properties with the spectral properties of the mapping matrix. The relation between the spectral properties of the mapping matrix and the mixing properties is demonstrated in Sec. V for an idealized timeperiodic flow. The potential for application to realistic industrial flows is demonstrated in Sec. VI. Conclusions are drawn in Sec. VII.

\section{EIGENMODE ANALYSIS OF ADVECTIVE(-DIFFUSIVE) SCALAR TRANSPORT}

The eigenmode analysis of distributive mixing presented in this study hinges on methodologies employed for eigenmode analyses of advective-diffusive scalar transport. Below a concise overview of the aspects of these analyses relevant in the present context is given.

\section{A. Systems with steady flow}

Advective-diffusive transport of a scalar quantity $C(\boldsymbol{x}, t)$ in the domain $\mathcal{D}$ is governed by

$$
\frac{\partial C}{\partial t}=L(C), \quad L=-\boldsymbol{u} \cdot \nabla+\frac{1}{\mathrm{Sc}} \nabla^{2}, \quad C(\boldsymbol{x}, 0)=C_{0}(\boldsymbol{x}),
$$

completed by steady linear conditions on boundary $\Gamma=\partial \mathcal{D}$. Here $L$ is the linear advection-diffusion operator, with leading and trailing terms representing advection and diffusion, respectively, $\boldsymbol{u}=\boldsymbol{u}(\boldsymbol{x})$ is the steady-flow field, and Sc $=U L / \kappa$ is the Schmidt number, where $U$ and $L$ are characteristic velocity and length scale, respectively, and $\kappa$ is the mass-diffusion coefficient. ${ }^{7}$ The velocity field is subject to the incompressibility constraint $\nabla \cdot \boldsymbol{u}=0$ and momentum conservation. Moreover, zero net flux of $C$ across boundary $\Gamma$ is assumed, implying conservation of the total scalar amount contained in the domain $\mathcal{D}$,

$$
M=\int_{\mathcal{D}} C(\boldsymbol{x}, t) d v=\int_{\mathcal{D}} C_{0}(\boldsymbol{x}) d v
$$

Solutions to Eq. (1) admit expansion into the form

$$
C(\boldsymbol{x}, t)=\sum_{k=0}^{\infty} \alpha_{k} H_{k}(\boldsymbol{x}, t), \quad H_{k}(\boldsymbol{x}, t)=e^{\mu_{k} t} \psi_{k}(\boldsymbol{x}),
$$

with $H_{k}$ as the eigenmodes dictating the spatiotemporal evolution of the scalar field. The corresponding eigenfunctioneigenvalue pairs $\left\{\psi_{k}, \mu_{k}\right\}$ are governed by the eigenvalue problem

$$
L\left(\psi_{k}\right)-\mu_{k} \psi_{k}=0,
$$

which follows readily from substitution of Eq. (3) into Eq. (1). Coefficients $\alpha_{k}$ are determined by the initial state $C_{0}(\boldsymbol{x})$ and constraint (2) implies one trivial eigenfunctioneigenvalue pair $\left\{\psi_{0}, \mu_{0}\right\}=\left\{c_{0}, 0\right\}$, with $c_{0}$ as a constant, and $\int_{\mathcal{D}} \psi_{k}(\boldsymbol{x}) d v=0$ for the nontrivial (inhomogeneous) eigenfunctions $\psi_{k}(\boldsymbol{x})(k \geq 1)$. Substitution of Eq. (3) into Eq. (2) yields $\sum_{k=0}^{\infty} \alpha_{k} e^{\mu_{k} t} \int_{\mathcal{D}} \psi_{k}(\boldsymbol{x}) d v=M$ for all $t \geq 0$. This constraint can be satisfied only if there exists one trivial eigenfunctioneigenvalue pair $\left\{\psi_{0}, \mu_{0}\right\}=\left\{c_{0}, 0\right\}$, with constant $c_{0}=M / \alpha_{0} V$ and $V=\int_{\mathcal{D}} d v$, in conjunction with nontrivial eigenfunctions $\psi_{k}(k \geq 1)$ meeting $\int_{\mathcal{D}} \psi_{k}(\boldsymbol{x}) d v=0$. Note $c_{0}$ depends on the initial distribution via $\alpha_{0}$. Resolving Eq. (4) for the specific boundary conditions and flow field $\boldsymbol{u}$ yields the particular nontrivial eigenfunction-eigenvalue pairs $\left\{\psi_{k}, \mu_{k}\right\}$-and thereby the particular inhomogeneous eigenmodes $H_{k}(\boldsymbol{x}, t)$ - for that transport problem.

The eigenvalues of the nontrivial eigenmodes are of the form $\mu_{k}=\sigma_{k}+i \omega_{k}$, with $\sigma_{k}$ and $\omega_{k}$ as real numbers, and possess the following generic properties relevant here: ${ }^{8,19}$

- $\sigma_{k} \leq 0$ due to the dissipative nature of the system;

- $\sigma_{k}<0$ for all inhomogeneous eigenmodes in case of bounded Sc;

- $\sigma_{k}=0$ for inhomogeneous eigenmodes is possible only for unbounded Sc.

Thus inhomogeneous eigenmodes in the case of advective-diffusive transport (bounded Sc) invariably exhibit exponential decay in time $\left(\sigma_{k}<0\right)$. This means that the evolution rapidly becomes dominated by the eigenmode with the slowest decay rate (i.e., with eigenvalue $\sigma_{k}$ closest to zero: $\left.\sigma_{\text {dom }}\right)$; this is the "dominant eigenmode." The scalar distribution quickly adopts the spatial distribution of the dominant eigenmode (with eigenvalue $\sigma_{\mathrm{dom}}$ ) and decays self-similarly towards its asymptotic state-defined by the homogenous eigenmode associated with Eq. (2) —with a characteristic time scale $\tau=1 /\left|\sigma_{\text {dom }}\right|$. [Self-similarity here means that the spatial distributions of the scalar field at various times can be obtained from one another via a similarity transformation or, equivalently, that the scalar field is of the form $C(\boldsymbol{x}, t)$ $=F(t) G(x) .{ }^{20}$ Hence, the spatial distributions of $C$ at different times identify up to a scaling factor.] This decay is monotonous and oscillatory for $\omega_{\mathrm{dom}}=0$ and $\omega_{\mathrm{dom}} \neq 0$, respectively. The spatial structure of the inhomogeneous eigenmodes becomes more intricate with growing Sc (diminishing diffusion increases scalar gradients and thus promotes spatial complexity); such spatially complex eigenmodes are denoted "strange eigenmodes.",2, Purely advective transport (unbounded Sc) admits, besides the above properties, inhomogeneous eigenmodes with $\sigma_{k}=0$ and thus may have inhomo- 
geneous asymptotic states. This sets advective transport fundamentally apart from advective-diffusive transport. The evolution toward its (in-)homogeneous asymptotic state is, essentially similar to the advective-diffusive case, also governed by one dominant eigenmode.

\section{B. Systems with two-dimensional time-periodic or three-dimensional spatially periodic steady flows}

Two-dimensional time-periodic and three-dimensional spatially periodic steady flows are flow fields with the structure

$$
\boldsymbol{u}(x, y, t)=\boldsymbol{u}(x, y, t+T), \quad \boldsymbol{u}(x, y, z)=\boldsymbol{u}(x, y, z+Z),
$$

respectively, where $T$ is the period time and $Z$ is the period length. Under the premise of unidirectional $u_{z}$, a condition usually met in duct flows (a class including the, in the present scope, relevant industrial inline mixers), the progression in the $z$-direction is equivalent to a progression in time, meaning that both types of flows are dynamically similar. In fact, three-dimensional spatially periodic steady flows with one unidirectional component can be transformed into twodimensional time-periodic flows $v \cdot v .^{22}$ Hence, the generic properties of the corresponding advective-diffusive scalar transport are in the remainder of this section exemplified in terms of two-dimensional time-periodic systems. These concepts are applied to both time-periodic and spatially periodic systems in Secs. III-VI.

Time periodicity in flow (and possibly also in the linear boundary conditions) causes the advection-diffusion operator $L$ in Eq. (1) to change constantly, meaning that the scalar transport can no longer be investigated in terms of the eigenmodes of $L$. However, a time-periodic counterpart exists in the Floquet operator $\mathcal{F}$, which maps the scalar distribution between discrete time levels $t=n T$, with $n \geq 0$ an integer, via

$$
C_{n+1}=\mathcal{F}\left(C_{n}\right), \quad C_{n}=\mathcal{F}^{n}\left(C_{0}\right), \quad C_{n}=C(\boldsymbol{x}, n T),
$$

and thus incorporates the integral effect of the advectiondiffusion operator (i.e., the total spatial redistribution of the scalar) within one period. The Floquet operator inherits the linear nature of the advection-diffusion operator, implying that the mapping by $\mathcal{F}$ permits an eigenmode decomposition similar to that of the steady system considered before,

$$
C_{n}(\boldsymbol{x})=\sum_{k=0}^{\infty} \gamma_{k} \mathcal{H}_{k}^{(n)}(\boldsymbol{x}), \quad \mathcal{H}_{k}^{(n)}(\boldsymbol{x})=\nu_{k}^{n} \Psi_{k}(\boldsymbol{x}),
$$

with $\mathcal{H}_{k}^{(n)}$ as the eigenmodes and $\left\{\Psi_{k}, \nu_{k}\right\}$ as the corresponding eigenfunction-eigenvalue pairs governed by the eigenvalue problem

$$
\mathcal{F}\left(\Psi_{k}\right)-\nu_{k} \Psi_{k}=0,
$$

and coefficients $\gamma_{k}$ determined by the initial state. ${ }^{8}$ Constraint (2) implies, similar to the steady case, one trivial eigenfunction-eigenvalue pair $\left\{\Psi_{0}, \nu_{0}\right\}=\left\{c_{0}, 1\right\}$, with $c_{0}$ as a constant, and $\int_{\mathcal{D}} \Psi_{k}(\boldsymbol{x}) d v=0$ for the nontrivial (inhomogeneous) eigenfunctions $(k \geq 1)$.

The inhomogeneous eigenmodes $\mathcal{H}_{k}^{(n)}$ determine the spatiotemporal evolution of the scalar distribution under timeperiodic conditions in a similar way as the eigenmodes $H_{k}$ in the steady-flow case. The eigenvalues $\nu_{k}$ may be complex and the properties relevant here are in essence time-periodic counterparts of those of eigenvalues $\sigma_{k}$ of the steady case:

- $\left|\nu_{k}\right| \leq 1$ due to the dissipative nature of the system;

- $\left|\nu_{k}\right|<1$ for all inhomogeneous eigenmodes in case of bounded Sc;

- $\left|\nu_{k}\right|=1$ for inhomogeneous eigenmodes is possible only for unbounded Sc.

The temporal evolution of the scalar distribution under time-periodic conditions thus has essential similarities with that under steady conditions. Advective-diffusive transport (bounded Sc) always leads to exponential decay toward a homogeneous asymptotic state; this decay, as in the steady case, is governed by a dominant (strange) eigenmode (i.e., corresponding with $\left|\nu_{k}\right|<1$ closest to one: $\nu_{\text {dom }}$ ) and occurs at a characteristic time scale $\tau=-T / \ln \left|\nu_{\text {dom }}\right|$. (Note that $\tau$ is the reciprocal of the dominant exponent $\Lambda$ introduced in Refs. 8 and 9.) Purely advective transport (unbounded Sc) displays the same behavior yet admits inhomogeneous asymptotic states (composed of the set of eigenmodes for which $\left|\nu_{k}\right|=1$ ). Essential difference between time-periodic and steady evolutions is that the former must not necessarily be self-similar. The evolution is self-similar if the corresponding eigenvalue of the dominant eigenmode is real; the evolution involves spatial redistribution of the scalar field if the corresponding eigenvalue of the dominant eigenmode is complex.

The connection between the eigenvalues/modes and the temporal evolution of the scalar distribution-and then specifically in relation to distributive mixing (unbounded Sc) — is elaborated in more detail in Sec. IV. To this end first the mapping method, an efficient method for the analysis of distributive mixing in time-periodic and spatially periodic systems, is introduced in Sec. III.

\section{THE MAPPING METHOD FOR DISTRIBUTIVE MIXING}

Chaotic mixing of viscous liquids in laminar flows is usually based on the situation where the baker's transformation is applied, a number of times on a specified volume of material. Reference 13 suggested that the distribution of material in such flows can be handled quite well by the use of matrix methods. The mapping method describes the transport of a conservative quantity from one state to another by means of a mapping matrix, describing the transport of fluid from an initial cross section to a final one (for spatially periodic flows) or from an initial time to a final time (for timeperiodic flows). Thus the mapping method concerns purely advective transport and, consequently, coincides with the transport systems in Sec. II for the limit $\mathrm{Sc} \rightarrow \infty$.

Recently we have formulated an alternative approach to compute the mapping matrix that is much simpler to implement ${ }^{23,24}$ as compared to the original approach. Figure 1 depicts how the entries of the mapping matrix are calculated in the new approach. To approximate the entries of the mapping (or distribution) matrix, $K$ markers are released inside each cell and tracked from $z=z_{0}$ to $z=z_{0}+\Delta z$. If the 


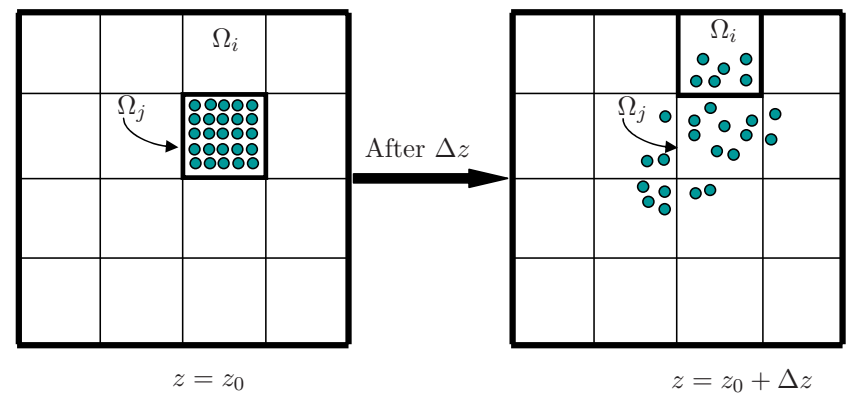

FIG. 1. (Color online) Illustration of the computation of the entries $\Phi_{i j}$ of the mapping matrix $\boldsymbol{\Phi}$. The cell $\Omega_{j}$ at $z=z_{0}$ is covered with a number of markers that are tracked during flow in $\Delta z$ (to arrive at the final cross section $z=z_{0}+\Delta z$ ). The ratio of the number of markers received by the recipient cell $\Omega_{i}$ to the initial number of markers in $\Omega_{j}$ is determined (in this example $\Phi_{i j}$ is $\left.\frac{6}{25}\right)$.

number of markers in the donor cell $\Omega_{j}$ is $M_{j}$ at $z=z_{0}$ and the number of markers found after tracking in the recipient cell $\Omega_{i}$ is $M_{i j}$ at $z=z_{0}+\Delta z$, then the entry $\Phi_{i j}$ is calculated as

$$
\Phi_{i j}=\frac{M_{i j}}{M_{j}}
$$

In other words, the entry $\Phi_{i j}$ is the measure of the fraction of the total flux of cell $\Omega_{j}$ donated to cell $\Omega_{i}$.

Once the mapping matrix $\boldsymbol{\Phi}$ is computed, the concentration vector $\boldsymbol{C} \in \mathbb{R}^{N \times 1}$ ( $N$ is the number of cells), after the one period of flow, $\boldsymbol{C}_{1}$, can be obtained by simply multiplying the mapping matrix $\boldsymbol{\Phi}$ with the initial concentration vector $\boldsymbol{C}_{0}$,

$$
C_{1}=\Phi C_{0}
$$

Vector $C$ represents the coarse-grained description of the volume fraction (i.e., the dimensionless concentration) of a passive marker in a fluid and each component $C^{(i)}$ describes the concentration (volume fraction) locally averaged in the associated cell $\Omega_{i}$. The relation in Eq. (10) describes the redistribution of the initial scalar distribution $\boldsymbol{C}_{0}$ at $t=0$ to its final state $\boldsymbol{C}_{1}$ at $t=T$ by the flow.

For repetitive mixing, the same operation is repeated multiple times on the same mass and, hence, the concentration evolution after $n$ steps is given by $\boldsymbol{C}_{n}=\boldsymbol{\Phi}^{n} \boldsymbol{C}_{0}$. For sufficiently large $n$, the matrix $\boldsymbol{\Phi}^{n}$ will not be sparse and it becomes that large that it can even not be stored anymore. This is due to the fact that after performing the operation $n$ times, material from one cell is advected to a large part of the whole cross section, especially in the case of chaotic advection. Instead of studying $\boldsymbol{\Phi}^{n}$, the evolution of the concentration after $n$ steps $\boldsymbol{C}_{n}$ is computed in sequence as follows:

$$
\boldsymbol{C}_{i+1}=\boldsymbol{\Phi} \boldsymbol{C}_{i} \text {, hence } \underbrace{\boldsymbol{C}_{n}=(\boldsymbol{\Phi}\{\boldsymbol{\Phi}}_{n \text { times }}\left[\ldots\left(\boldsymbol{\Phi} \boldsymbol{C}_{0}\right)\right]\})
$$

Thus, the mapping matrix $\boldsymbol{\Phi}$ is determined only once and is utilized a number of times to study the evolution of concentration in the flow field.

\section{EIGENMODE ANALYSIS OF DISTRIBUTIVE MIXING}

\section{A. The mapping matrix versus the Floquet operator}

The mapping matrix $\boldsymbol{\Phi}$ according to Eq. (10) describes the advective redistribution of a scalar quantity over a finite number of cells during one period in a periodic system and thus constitutes a discrete approximation to the Floquet operator $\mathcal{F}$ according to Eq. (6) for unbounded Sc.

The mapping $\boldsymbol{C}_{n}=\boldsymbol{\Phi}^{n} \boldsymbol{C}_{0}$ (employing $N$ cells) admits the eigenmode decomposition

$$
\boldsymbol{C}_{n}=\boldsymbol{\Phi}^{n} \boldsymbol{C}_{0}=\sum_{k=0}^{N-1} \widetilde{C}_{k}^{0} \boldsymbol{h}_{k}^{(n)}, \quad \boldsymbol{h}_{k}^{(n)}=\lambda_{k}^{n} \boldsymbol{v}_{k}, \quad \boldsymbol{C}_{0}=\sum_{k=0}^{N-1} \widetilde{C}_{k}^{0} \boldsymbol{v}_{k},
$$

with $\boldsymbol{h}_{k}^{(n)}$ as the eigenmodes and $\left\{\boldsymbol{v}_{k}, \lambda_{k}\right\}$ as the corresponding eigenvector-eigenvalue pairs of $\boldsymbol{\Phi}$ and coefficients $\widetilde{C}_{k}^{0}$ determined by the initial state $\boldsymbol{C}_{0}$. Vector-scalar pairs $\left\{\boldsymbol{v}_{k}, \lambda_{k}\right\}$ and vectors $\boldsymbol{h}_{k}^{(n)}$ are the discrete approximations to the eigenfunction-eigenvalue pairs $\left\{\Psi_{k}, \nu_{k}\right\}$ of the Floquet operator and the eigenmodes $\mathcal{H}_{k}^{(n)}$ in Eq. (7), respectively. Here we assume that the mapping method is consistent in the sense that the action of the mapping matrix converges on that of the continuous Floquet operator with increasing spatial resolution (i.e., increasing number of cells $N$ ). Under this premise, the mapping matrix is indeed a discrete approximation to the Floquet operator. Although rigorous proof is unavailable (and beyond the present scope), numerical studies strongly suggest this assumption to hold true. ${ }^{25}$ This assumption, in turn, implies that the spectral decomposition of the mapping matrix is a discrete approximation to that of the Floquet operator. Within the scope of this study we furthermore assume that the number of cells $(N)$ is such that the discrete operators and quantities provide sufficiently accurate approximations of their continuous counterparts. The properties of the eigenfunction-eigenvalue pairs of the Floquet operator due to constraint (2) translate into a trivial eigenvector-eigenvalue pair $\left\{\boldsymbol{v}_{0}, \lambda_{0}\right\}=\left\{c_{0} \mathbf{1}, 1\right\}$, with $\mathbf{1}$ as the unit vector and $c_{0}$ again as a constant, and $\sum_{i=0}^{N-1} v_{k, i}=0$ for the nontrivial (inhomogeneous) eigenvectors $\boldsymbol{v}_{k}(k \geq 1)$.

Thus a direct connection between the mapping matrix and the eigenmode decomposition (7) has been established. The mapping matrix incorporates the advection process and, consequently, all information on the transport properties is "hidden" in its eigenvalue-eigenvector pairs in the same manner as discussed before for the eigenvalue-eigenfunction pairs of the Floquet operator $\mathcal{F}$ (Sec. II). Eigenmode analyses have thus far sought to investigate the evolution of scalar distributions toward the homogeneous asymptotic state for advective-diffusive transport (bounded Sc). The present work seeks to pursue a similar course of action for the investigation of distributive mixing (unbounded Sc) in twodimensional time-periodic and three-dimensional spatially periodic steady systems. To this end the properties of the eigenvalue-eigenvector pairs of the mapping matrix must be tied specifically to distributive mixing. This is elaborated below. 


\section{B. Generic advection properties}

The mapping matrix is real for physically meaningful problems, meaning that complex eigenvalues and associated eigenvectors emerge as conjugate pairs $\left\{\lambda_{k}, \lambda_{k}^{*}\right\}$ and $\left\{\boldsymbol{v}_{k}, \boldsymbol{v}_{k}^{*}\right\}$, respectively. The contributions of complex-conjugate pairs $\left\{\boldsymbol{v}_{k}, \boldsymbol{v}_{k+1}\right\}=\left\{\boldsymbol{v}_{k}, \boldsymbol{v}_{k}^{*}\right\}$ to the initial conditions $\boldsymbol{C}_{0}$ read

$$
\begin{aligned}
\boldsymbol{C}_{0}= & \cdots+\widetilde{C}_{k}^{0} \boldsymbol{v}_{k}+\widetilde{C}_{k+1}^{0} \boldsymbol{v}_{k+1}+\cdots=\cdots+\widetilde{C}_{k}^{0} \boldsymbol{v}_{k}+\widetilde{C}_{k+1}^{0} \boldsymbol{v}_{k}^{*} \\
& +\cdots,
\end{aligned}
$$

which, by virtue of real $\boldsymbol{C}_{0}$, must be real. This implies real and pairwise identical expansion coefficients for complexconjugate pairs in the initial state, i.e., $\widetilde{C}_{k}^{0}=\widetilde{C}_{k+1}^{0}$, and resulting contributions following

$$
\boldsymbol{C}_{0}=\cdots+\widetilde{C}_{k}^{0}\left[\boldsymbol{v}_{k}+\boldsymbol{v}_{k}^{*}\right]+\cdots=\cdots+2 \widetilde{C}_{k}^{0} \operatorname{Re}\left(\boldsymbol{v}_{k}\right)+\cdots,
$$

meaning complex-conjugate pairs of eigenvectors effectively constitute one physically meaningful eigenvector. The eigenmode decomposition (12) thus effectively is of the form

$$
\begin{aligned}
\boldsymbol{C}_{n}= & \boldsymbol{\Phi}^{n} \boldsymbol{C}_{0}=\cdots+\widetilde{C}_{k}^{0} \lambda_{k}^{n} \boldsymbol{v}_{k}+\cdots \\
& +2 \widetilde{C}_{k}^{0}\left|\lambda_{k}\right|^{n}\left[\begin{array}{c}
V_{k, 0} \cos \left(\alpha_{k, 0}+n \phi_{k}\right) \\
\vdots \\
V_{k, N} \cos \left(\alpha_{k, N}+n \phi_{k}\right)
\end{array}\right]+\cdots,
\end{aligned}
$$

with leading and trailing terms on the right hand side representing contributions by real and complex eigenmodes, respectively, where $V_{k, i}=\left|v_{k, i}\right|, \alpha_{k, i}=\arg \left(v_{k, i}\right)$, and $\phi_{k}=\arg \left(\lambda_{k}\right)$.

The structure of mapping (15) reveals that its action on nontrivial real eigenmodes consists solely of periodwise rescaling by a constant factor $\left|\lambda_{k}\right| \leq 1$; its action on nontrivial complex eigenmodes consists, besides this rescaling, of spatial redistribution due to the phase shift $\phi_{k}$. Hence, nontrivial real eigenmodes always evolve self-similarly in the sense defined before. [The discrete analogon to the form $C(\boldsymbol{x}, t)$ $=F(t) G(\boldsymbol{x})$ given above reads $\boldsymbol{C}(n)=F(n) \boldsymbol{G}$.] Nontrivial complex eigenmodes, on the other hand, evolve self-similarly only for specific $\phi_{k}$. Eigenmodes with self-similar evolution play a pivotal role in the asymptotic advection pattern. This is discussed in Sec. IV C.

The eigenvalue spectrum of $\boldsymbol{\Phi}$ yields both qualitative and quantitative insights into the generic advection properties. The eigenvalues of inhomogeneous eigenmodes all meeting $\left|\lambda_{k}\right|<1$ implies progression toward a homogeneous ("well-mixed") asymptotic state defined by the trivial eigenmode; conversely, at least one of these eigenvalues meeting $\left|\lambda_{k}\right|=1$ implies persistent inhomogeneities in the asymptotic state. Hence, $\left|\lambda_{k}\right|<1$ for all inhomogeneous eigenmodes is a qualitative "good-mixing" criterion. The progression toward the (in-)homogeneous asymptotic state is dominated by the eigenmode $\boldsymbol{h}_{k}^{(n)}$ with the highest $\left|\lambda_{k}\right|<1$ (i.e., $\lambda_{\text {dom }}$ ) and occurs with characteristic time scale $\tau_{\lambda}=-T / \ln \left|\lambda_{\text {dom }}\right|$, where $\lambda_{\text {dom }}$ and $\tau_{\lambda}$ are the discrete approximations to the dominant eigenvalue $\nu_{\mathrm{dom}}$ and characteristic decay time $\tau$, respectively, defined in Sec. II B. For good-mixing cases, $\tau_{\lambda}$ thus is the characteristic homogenization time and thereby a quantita- tive mixing measure. Initial inhomogeneities are, e.g., reduced to less than $1 \%$ within a time interval $\Delta t=5 \tau_{\lambda}$. Note that in case of multiple eigenmodes "competing" for dominance due to closeness of associated eigenvalues, the duration of the homogenization process remains determined by $\tau_{\lambda}$; such competition manifests itself solely in the intermediate spatial redistribution.

\section{Properties of the asymptotic advection pattern}

The properties of the mapping matrix, in addition to the above generic properties, also afford insight into the (in)homogeneous asymptotic advection pattern. This connection is heuristically established by physical rationales leaning on findings from mixing studies on two-dimensional timeperiodic incompressible flows rather than on rigorous mathematical grounds. (The latter approach is highly nontrivial and beyond the present scope.) From mixing studies on said flows it is well known that the asymptotic advection pattern is determined by periodic points, i.e., material points that systematically return to their initial position after a given number of periods, and their associated spatial structures. ${ }^{26-30}$ In three-dimensional spatially periodic systems according to Eq. (5) these periodic points and structures emerge in the two-dimensional mapping between spatial levels $[0, Z, 2 Z, \ldots] .^{22}$

Incompressible flows admit two types of nondegenerate periodic points: (i) elliptic points, which form the center of persistent nonmixing regions ("elliptic islands"); (ii) hyperbolic points, the associated manifolds of which accomplish the exponential stretching and folding of fluid parcels that underlies chaotic advection. ${ }^{29}$ Generic two-dimensional advection patterns associated with periodic systems following Eq. (5) thus consist of elliptic islands embedded in chaotic seas created by manifolds. This generic picture forms the basis for the connection between the properties of the mapping matrix $\boldsymbol{\Phi}$ and the asymptotic advection pattern established hereafter.

Periodic points and their associated spatial structures appear in the mapping as fixed entities or as cycles. This evolution manifests itself in mapping (15) as phase shifts $\phi$ commensurate with $2 \pi$ and, consequently, eigenvalues of the form

$$
\lambda=|\lambda| e^{2 \pi i m / p},
$$

for the corresponding eigenmodes, with $p$ as the periodicity and $0 \leq m \leq p-1$ as an arbitrary integer. (The subscript $k$ is omitted for brevity.) The eigenmodes themselves satisfy

$$
\boldsymbol{h}^{(n+p)}=\boldsymbol{\Phi}^{p} \boldsymbol{h}^{(n)}=|\lambda|^{p} \boldsymbol{h}^{(n)},
$$

meaning that a period $p$ eigenmode is self-similar with every $p$ th mapping of itself. Note that periodicity, somewhat unconventionally, is determined solely by the evolution in time; the corresponding eigenmode may persist $(|\lambda|=1)$ or decay $(|\lambda|<1)$ in time. Liu and Haller ${ }^{2}$ employed essentially the same nomenclature by denoting strange eigenmodes with evolution commensurate to the period time also as time periodic. The asymptotic advection pattern thus, since it is determined by periodic structures, must comprise entirely of 
periodic eigenmodes according to Eq. (17). Behavior known from mixing studies implies that these periodic eigenmodes must possess properties according to the conjectures below.

Conjecture 1. Elliptic islands relate to persistent $(|\lambda|$ =1) eigenmodes according to Eq. (17).

Rationale: This follows readily from the fact that elliptic islands give rise to persistent inhomogeneities in the asymptotic scalar distribution.

Conjecture 2. Chaotic seas relate to dominant $(|\lambda|<1)$ eigenmodes according to Eq. (17).

Rationale: Chaotic seas are characterized by exponential convergence of scalar distributions onto the unstable manifold(s) of the corresponding hyperbolic periodic point(s), resulting in intricate advection patterns with a highly complex (i.e., "strange") spatial structure. This is consistent with the exponential decay effectuated by a dominant (strange) eigenmode.

Conjecture 3. Eigenmodes associated with elliptic islands and chaotic seas must be spatially disconnected. To this end eigenmodes must not affect the spatiotemporal scalar evolution outside their associated spatial entities.

Rationale: Elliptic islands result in persistent inhomogeneities; chaotic seas cause exponential decay of scalar distributions. Eigenmodes can thus either belong to islands $(|\lambda|$ $=1)$ or to chaotic seas $(|\lambda|<1)$ yet not to both entities simultaneously. Hence, eigenmodes associated with islands (chaotic seas) must not contribute to the spatial redistribution of material in the region outside these islands (chaotic seas). This selective contribution to the transport properties implies the spatial disconnection according to Conjecture 3 .

The above, in general, requires periodic eigenmodes (and thus also its underlying eigenvectors) to vanish in the regions outside their corresponding islands or chaotic seas. The sole exception to this rule is periodic eigenmodes with $\lambda=1$ (belonging to elliptic islands); they must only be homogeneous outside their corresponding island(s) so as not to contribute to the spatiotemporal evolution there. The regions inside and outside spatial entities are henceforth denoted the "active" and "inactive" zones of the associated eigenmodes. Recall to this end that the vector entries in the mapping representation correspond to an area ("cell") in the domain of interest. Hence, (in-)active zone regions correspond to specific matrix/vector entries.

The natural consequence of Conjecture 3 is that spatial entities in the asymptotic advection pattern of the same periodicity and kind (i.e., persistent or decaying) are inextricably linked with a given set of eigenvector-eigenvalue pairs $\{\boldsymbol{v}, \lambda\}$. This is elaborated further in the following:

Conjecture 4. Period-p structures of equal kind correspond with clusters of eigenvalues

$$
\Lambda=\left\{\lambda^{(0)}, \lambda^{(1)}, \ldots, \lambda^{(m)}, \ldots, \lambda^{(p-1)}\right\},
$$

with $\lambda^{(m)}=|\lambda| \omega^{m}$ and $\omega=e^{2 \pi i / p}$.

Rationale: Expression (17) sets forth $\boldsymbol{\Phi}^{p} \boldsymbol{v}=|\lambda|^{p} \boldsymbol{v}$ as a relation for the eigenvector-eigenvector pairs $\{\boldsymbol{v}, \lambda\}$ of $\boldsymbol{\Phi}$ associated with period $p$ eigenmodes. Matrices $\boldsymbol{\Phi}^{p}$ and $\boldsymbol{\Phi}$ share the same eigenvectors; eigenvalues relate via $\operatorname{eig}\left(\boldsymbol{\Phi}^{p}\right)$ $=\operatorname{eig}(\boldsymbol{\Phi})^{p} \cdot{ }^{31}$ The cluster of eigenvalues (18) comprises the $p$ complex roots of $|\lambda|^{p}$, equidistantly distributed along the circle with radius $|\lambda|$ in the complex plane that satisfy this relation. ${ }^{32}$ This is consistent with the findings of mixing studies, which hold that period $p$ points $\boldsymbol{x}^{(0)}=\boldsymbol{F}^{p}\left(\boldsymbol{x}^{(0)}\right)$, with $\boldsymbol{x}^{(m)}$ $=\boldsymbol{F}^{m}\left(\boldsymbol{x}^{(0)}\right)$ and $\boldsymbol{F}$ as the mapping of material points, always form period $p$ structures comprising clusters of $p$ points $\mathcal{X}$ $=\left\{\boldsymbol{x}^{(0)}, \boldsymbol{x}^{(1)}, \ldots, \boldsymbol{x}^{(m)}, \ldots, \boldsymbol{x}^{(p-1)}\right\}$ and attendant spatial entities, $^{27,33}$ (island chains, e.g., encompass $p$ islands that each are centered on one of the points belonging to a cluster of $p$ elliptic period $p$ points), hence Conjecture 4 .

Conjecture 4 connects spatial entities with eigenvalues of $\boldsymbol{\Phi}$ in that a given period $p$ structure corresponds with one cluster (18); more generally, multiple period $p$ structures of the same kind result in repeated clusters (18). Each eigenvalue $\lambda^{(m)}$ in such (repeated) clusters has its (in principle distinct) eigenvector $\boldsymbol{v}_{j}^{(m)}$, with $1 \leq j \leq J$ indicating the $j$ th of in total $J$ periodic structures. This advances the cluster of eigenvector-eigenvalue pairs

$$
\begin{aligned}
\mathcal{S}= & \left\{\left\{\boldsymbol{v}_{1}^{(0)}, \lambda^{(0)}\right\}, \ldots,\left\{\boldsymbol{v}_{1}^{(p-1)}, \lambda^{(p-1)}\right\}, \ldots,\left\{\boldsymbol{v}_{j}^{(0)}, \lambda^{(0)}\right\},\right. \\
& \left.\ldots,\left\{\boldsymbol{v}_{j}^{(p-1)}, \lambda^{(p-1)}\right\}, \ldots,\left\{\boldsymbol{v}_{J}^{(0)}, \lambda^{(0)}\right\}, \ldots,\left\{\boldsymbol{v}_{J}^{(p-1)}, \lambda^{(p-1)}\right\}\right\},
\end{aligned}
$$

as combined "spectral footprint" of a group of $J$ period $p$ structures of the same kind ("period $p$ group") in the spectral decomposition of $\boldsymbol{\Phi}$ (e.g., $J=2, p=4$, and $|\lambda|=1$ for two period-4 island chains). The eigenvectors $\boldsymbol{v}_{j}^{(m)}$ play the following role:

Conjecture 5. The active zones of all eigenvectors $\boldsymbol{v}_{j}^{(m)}$ in Eq. (19) combined demarcate the invariant region occupied by the entire period $p$ group in the asymptotic advection pattern.

Rationale: From mixing studies it is known that each individual entity within period $p$ structures of a period $p$ group (e.g., individual islands in island chains) is mapped along the positions of its companion entities before returning to its initial position after $p$ periods. This implies that, since every entity undergoes this mapping, the region occupied by the period $p$ group as a whole is invariant. The distribution of quantity $C(\boldsymbol{x}, t)$ within this region must not necessarily remain constant. (For instance, island chains as a whole are fixed yet material in its constituent islands wanders along the chain during successive mappings.) This, upon reconciliation with the mapping method, implies that the combined active zones of all associated eigenvectors must demarcate this invariant region.

Important to note is that the contribution of individual eigenvectors $\boldsymbol{v}_{j}^{(m)}$ to the demarcation following Conjecture 5, saving the case of isolated period-1 structures, cannot be predicted beforehand. The forthcoming case studies in Secs. $\mathrm{V}$ and VI in fact demonstrate that these individual contributions may take several forms.

The conjectures advanced above permit the following connection between coherent structures in the asymptotic advection pattern and eigenmodes of the mapping matrix $\boldsymbol{\Phi}$ :

- Period $p$ island chains correspond to (repeated) clusters of eigenvalues $\left\{1, \omega, \omega^{2}, \ldots, \omega^{p-1}\right\}$ on the unit circle, where $\omega=e^{2 \pi i / p}$;

- Chaotic seas are created by clusters of period $p$ hyper- 


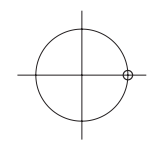

(a)

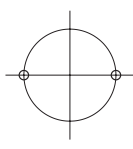

(b)

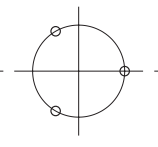

(c)

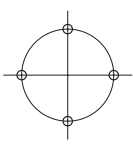

(d)

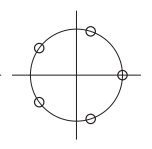

(e)

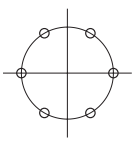

(f)
FIG. 2. Clusters of eigenvalues according to Eq. (18) for periodicities 1 $\leq p \leq 6$.

bolic points and associated manifolds that correspond to (repeated) clusters of eigenvalues $|\lambda|\left\{1, \omega, \omega^{2}, \ldots, \omega^{p-1}\right\}$ on the circle with radius $|\lambda|$ $<1$ and an identical eigenmode $\boldsymbol{v}$, where again $\omega$ $=e^{2 \pi i / p}$.

The corresponding eigenvectors combined demarcate the region(s) occupied by the island and chaotic seas in accordance with Conjecture 5. The exponential decay for eigenmodes with $|\lambda|<1$ implies that each spatially disconnected chaotic sea has its own dominant (strange) eigenmode of period $p$. However, global chaos implies one global (cluster of) dominant (strange) eigenmode(s). The corresponding (cluster) of eigenvalues lie(s) on the circle $\left|\lambda_{\text {dom }}\right|$ enclosing all other eigenvalues.

Figure 2 schematically depicts the circular arrangement of clusters of eigenvalues according to Eq. (18) in the complex plane for periodicities $1 \leq p \leq 6$. The circle coincides with the unit circle in case of islands chains and on the circle with radius $|\lambda|<1$ in case of chaotic seas. Shown arrangements reveal that any cluster includes eigenvalue $\lambda /|\lambda|=1$, clusters with even periodicity always include $\lambda /|\lambda|= \pm 1$, and even clusters commensurate with four $(p=4,8, \ldots)$ always include $\lambda /|\lambda|= \pm 1$ and $\lambda /|\lambda|= \pm i$. Moreover, period-1 and period-2 structures always coincide with real eigenmodes.

Sections V and VI demonstrate the link between asymptotic advection patterns and periodic eigenmodes of the mapping matrix $\boldsymbol{\Phi}$ by way of representative case studies.

\section{ILLUSTRATIVE EXAMPLE: THE TIME-PERIODIC SINE FLOW}

\section{A. Asymptotic advection patterns and corresponding eigenvalue spectra}

the time-periodic sine flow (TPSF) is a two-dimensional time-periodic flow system that may serve as an idealized representation of realistic mixing flows. ${ }^{8,34,35}$ The TPSF is set up by the periodic repetition of two piecewise steady flows according to

$$
\begin{aligned}
& \left(u_{x}, u_{y}\right)=[\sin (2 \pi y), 0] \quad \text { for } 0 \leq t \leq \frac{T}{2}, \\
& \left(u_{x}, u_{y}\right)=[0, \sin (2 \pi x)] \quad \text { for } \frac{T}{2} \leq t \leq T,
\end{aligned}
$$

on the spatially periodic domain $0 \leq x, y \leq 1$, where $T$ is the period time of the flow and $u_{x}$ and $u_{y}$ are the $x$ - and $y$-wise velocity components, respectively. (Note that the TPSF satisfies the incompressibility constraint.) The simple structure

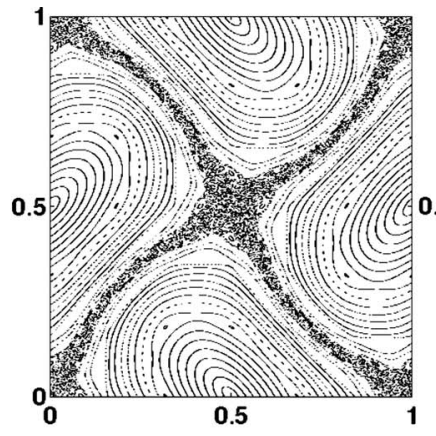

(a)

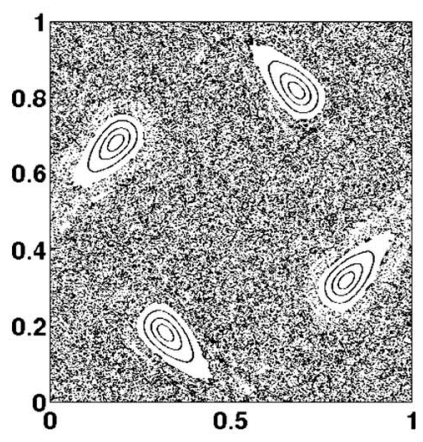

(c)

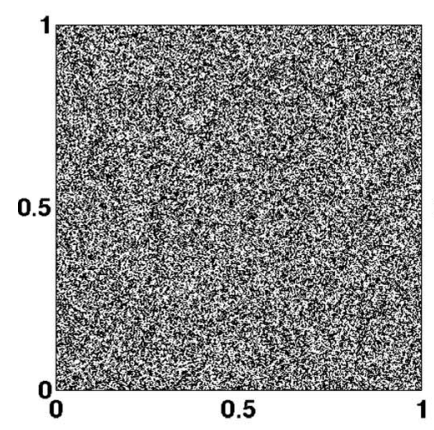

(e)

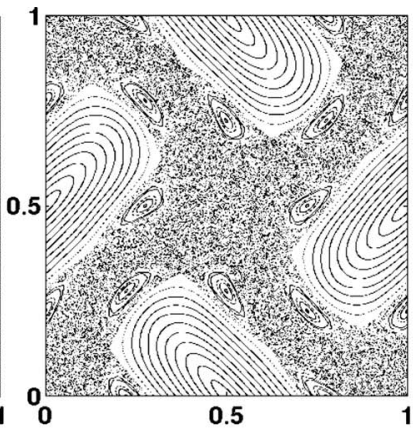

(b)

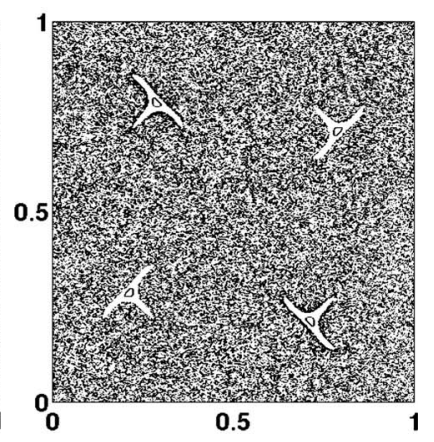

(d)

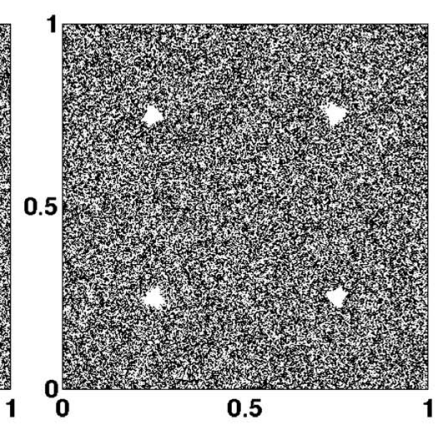

(f)
FIG. 3. Dynamics of the TPSF as a function of the period time $T$ in terms of Poincaré sections: (a) $T=0.4$; (b) $T=0.56$; (c) $T=0.8$; (d) $T=1.18$; (e) $T$ $=1.6$; (f) $T=3$.

of the TPSF admits an analytical expression for the fluid trajectories, reading

$$
\begin{aligned}
& x_{i}=\bmod \left[\left\{x_{i-1}+\frac{T}{2} \sin \left(2 \pi y_{i-1}\right)\right\}, 1\right], \\
& y_{i}=\bmod \left[\left\{y_{i-1}+\frac{T}{2} \sin \left(2 \pi x_{i}\right)\right\}, 1\right],
\end{aligned}
$$

with $i$ indicating the position after $i$ periods.

The period time $T$ is the only control parameter of the TPSF and its variation leads to different dynamics. ${ }^{8}$ This is demonstrated in Fig. 3 in terms of the Poincaré section of the flow, revealing that changing the period time from $T=0.4$ to $T=1.6$ has significant effects on the transport properties of the flow. Nonmixing zones (i.e., islands comprising regular closed orbits) first diminish with rising $T$ [panels (a)-(d)] until a state of global chaos-or efficient mixing — is attained 


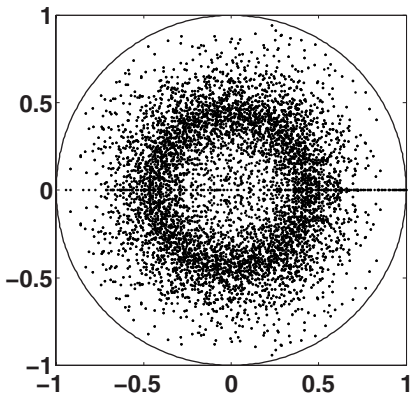

(a)

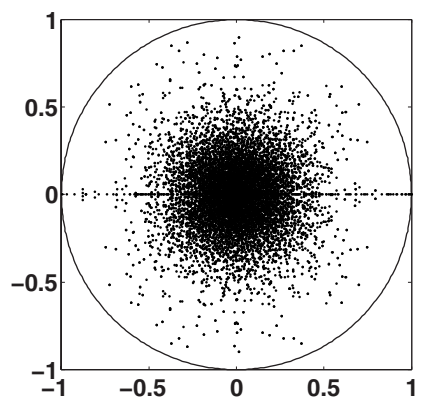

(c)

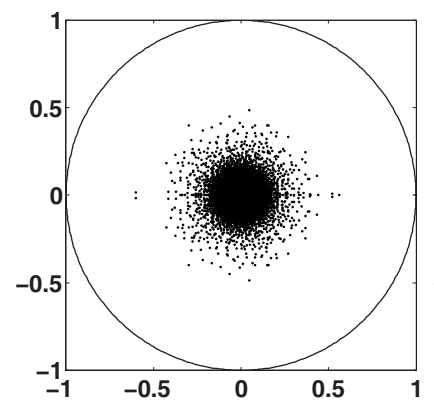

(e)

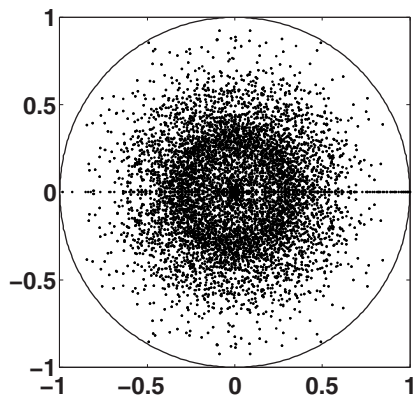

(b)

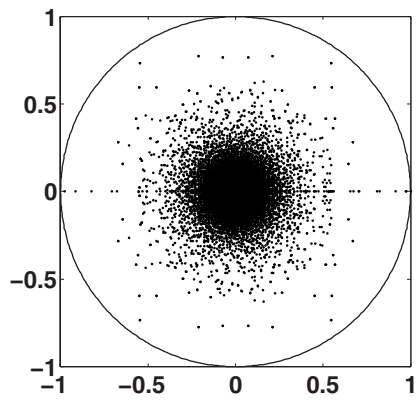

(d)

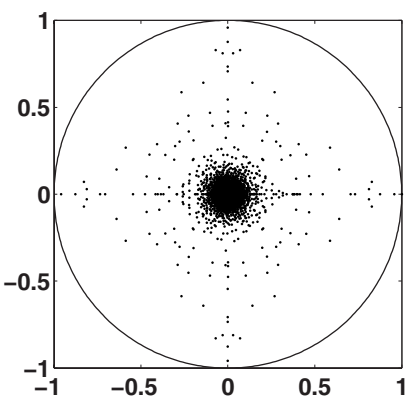

(f)
FIG. 4. Eigenvalue spectrum of the mapping matrix associated with the TPSF for the cases given in Fig. 3: (a) $T=0.4$; (b) $T=0.56$; (c) $T=0.8$; (d) $T=1.18$; (e) $T=1.6$; (f) $T=3$.

at $T=1.6[$ panel (e)] and reappear again upon further increasing $T$ [panel (f)]. This signifies a nonlinear dependence of the transport on the governing control parameter, which is typical of realistic mixing problems. The transport properties of the TPSF serve as test case for the eigenmode analysis of distributive mixing.

Figure 4 shows the eigenvalue spectra of the mapping matrices corresponding with the cases shown in Fig. 3. (The mapping matrices are evaluated using $100 \times 100$ cells.) The partially chaotic cases [Figs. 4(a)-4(d) and 4(f)] accommodate islands and, consistent with Conjecture 1, have (clusters of) eigenvalues on the unit circle. Note that the finite resolution of the mapping method in fact causes these eigenvalues to fall slightly within the unit circle due to numerical diffusion (Sec. V D).

The globally chaotic case $T=1.6$ [Fig. 4(e)] has, in agreement with Conjecture 2, an eigenvalue spectrum entirely within the unit circle, saving the eigenvalue $\lambda=1$ corresponding to the trivial eigenvector. Hence, eigenvalues on the unit circle other than the trivial one are a first qualitative indicator of poor mixing. Thus the spectral decomposition of the mapping matrix readily yields the gross mixing properties of a given flow. The relation between spectral decomposition and mixing properties is explored in more detail below.

\section{B. Eigenmode analysis of globally chaotic mixing}

Period time $T=1.6$ effectuates global chaos and, in accordance with Conjecture 2, has an eigenvalue spectrum residing entirely within the unit circle [Fig. 4(e)]. The global nature of the chaotic state implies one global (cluster of) dominant eigenmode(s) (Sec. IV). Here this state is dictated by one period-2 pair of dominant eigenmodes with associated eigenvalues $\Lambda=\lambda_{\text {dom }}\{1,-1\}$ conform Conjecture 19, where $\lambda_{\text {dom }} \approx 0.58$. The dominant eigenvalues are indicated by the small circles in the eigenvalue spectrum in Fig. 5(a) [compare to Fig. 2(b)] and span a circle with radius $\lambda_{\text {dom }}$ enclosing all other nontrivial eigenvalues. Note the dominant eigenvalue $\lambda=-\lambda_{\text {dom }}$ in fact appears as a complex-conjugate pair with very small imaginary part in the computed spectrum. Numerical experiments strongly suggest this to result from the finite resolution; the imaginary part namely diminishes-implying convergence of the complexconjugate pair on one real eigenvalue-with increasing resolution. Figures 5(b) and 5(c) give eigenvectors corresponding to $\lambda=-\lambda_{\text {dom }}$ and $\lambda=\lambda_{\text {dom }}$, respectively, of which at least one is nonzero in any given position. The eigenvectors associated with the complex-conjugate pair of eigenvalues representing the real dominant eigenvalue $\lambda=-\lambda_{\text {dom }}$ have, consistent with their associated eigenvalues, very small imaginary contributions that diminish with increasing resolution. Hence, shown eigenvector in fact corresponds with the physically meaningful (and identical) real parts of these eigenvectors. Thus the combined active zones of the dominant pair, consistent with Conjecture 5 , indeed demarcate the entire chaotic sea.

The essential role of the dominant pair of eigenmodes in the advection process is demonstrated in Fig. 6, which shows the evolution of an initially inhomogeneous scalar distribution: $C=0$ and $C=1$ below and above the line $y=1 / 2$, respectively [panel (a)]. Panels (b)-(h) show the subsequent evolution, in which the rapid convergence on the period-2 evolution dictated by the pair of dominant eigenmodes is clearly visible. The spatial distribution quickly develops the spatial features of the eigenmodes (Fig. 5) and the periodwise reversal of dark and bright regions-resulting in a selfsimilar decay every second period-nicely reflects the period-2 nature of the evolution. Note that the asymptotic advection pattern is in excellent agreement with that found in Refs. 5, 6, 8, and 9 via spectral analysis of the advectiondiffusion operator. Figure 6(i) gives the decay of the local concentration, defined as

$$
\delta(x, t)=\frac{\Delta C(x, t)}{\Delta C(x, 0)}, \quad \Delta C(x, t)=C(x, t)-C_{\infty},
$$

with $C_{\infty}=\int C_{0}(\boldsymbol{x}) d v$ as the asymptotic homogeneous state (here $C_{\infty}=0.5$ ), in a sequence of positions on the line $y$ $=3 / 4$ (markers) compared against the predicted homogenization time $\tau_{\lambda}=-T / \ln \lambda_{\mathrm{dom}}=2.94$ on the basis of the dominant 


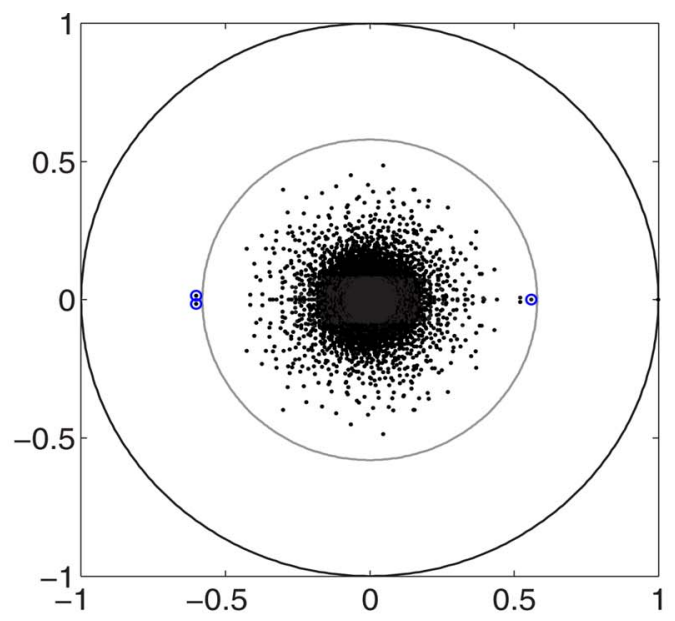

(a)

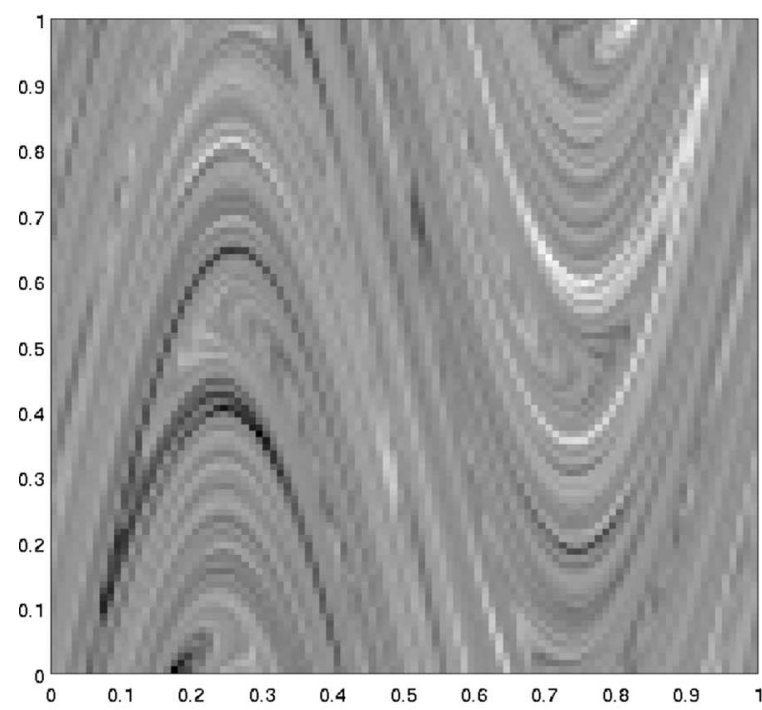

(b)
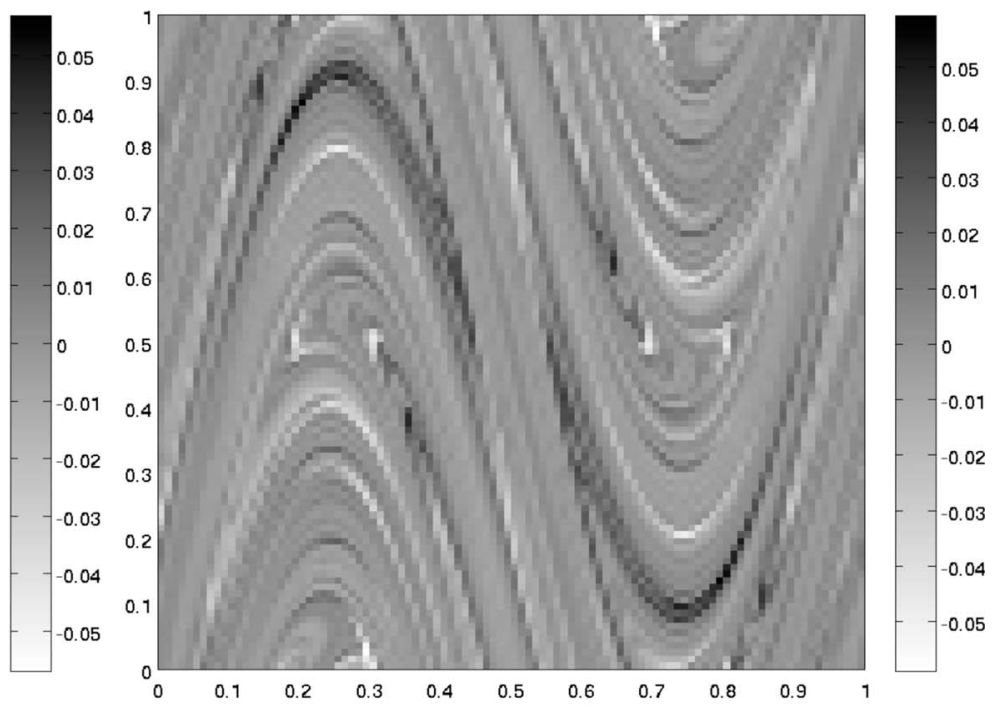

(c)

FIG. 5. (Color online) Period-2 pair of dominant eigenmodes for the globally chaotic TPSF ( $T=1.6)$ : (a) eigenvalue spectrum (period-2 cluster: small circles); (b) eigenvector for $\lambda=-\lambda_{\text {dom }}$; (c) eigenvector for $\lambda=\lambda_{\text {dom }}$.

eigenvalues (sloping line). The simulated decay rates are in excellent agreement with the prediction, thus substantiating the claim in Sec. IV B that the eigenvalue spectrum may indeed yield quantitative mixing characteristics.

The above findings permit optimization of the mixing process on the basis of the properties of the dominant eigenmode. Consider to this end the globally chaotic states at $T$ $=1.5, T=1.7, T=1.8$, and $T=1.9$ in the direct proximity of the case $T=1.6$. Poincare sections give indistinguishable results for all five cases (i.e., a picture of a globally chaotic state) and therefore fail to identify the most efficient one. However, the corresponding eigenvalue spectra (Fig. 7) reveal that the eigenvalues are most densely concentrated around the origin - and thus the dominant eigenvalue $\lambda_{\text {dom }}$ is minimal-for $T=1.8$, exposing this as the most efficient mixing case. Figures 8(a) and 8(b) show $\lambda_{\text {dom }}$ and the corresponding characteristic homogenization time $\tau_{\lambda}$, respectively, in the range $1.5 \leq T \leq 1.9$, quantifying the mixing ability of the chaotic flows and also putting forward $T=1.8$ as most efficient case. This further demonstrates the potential of the spectral decomposition of the mapping matrix for quantitative mixing analyses.

\section{Eigenmode analysis of partially chaotic mixing}

Partially chaotic mixing implies islands embedded in one (or more) chaotic sea(s). Cases $T=0.4,0.56$ and cases $T=0.8,1.18$, for instance, accommodate two period-1 islands and two period-2 island chains, respectively, embedded in one chaotic sea (Fig. 3). The eigenmode analysis of such partially chaotic systems is exemplified for that of the case $T=0.8$. The two period- 2 island chains correspond, compliant with Conjecture 19, to two period-2 clusters $\Lambda=\{1,-1\}$ on the real axis [compare to Fig. 2(b)]. The associated eigenvectors of the period-2 group according to Eq. (19) are given in Fig. 9: panels (a) and (b) and (c) and (d) correspond to 


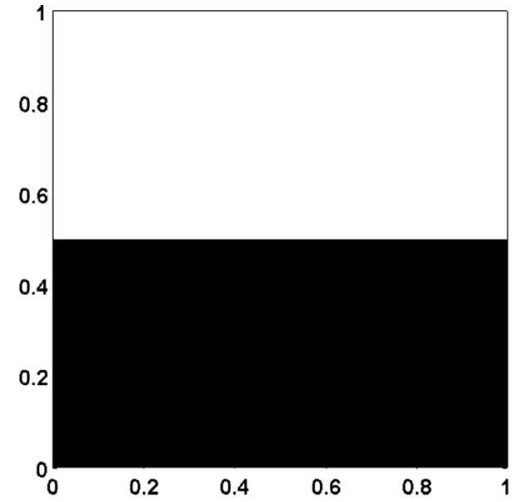

(a)

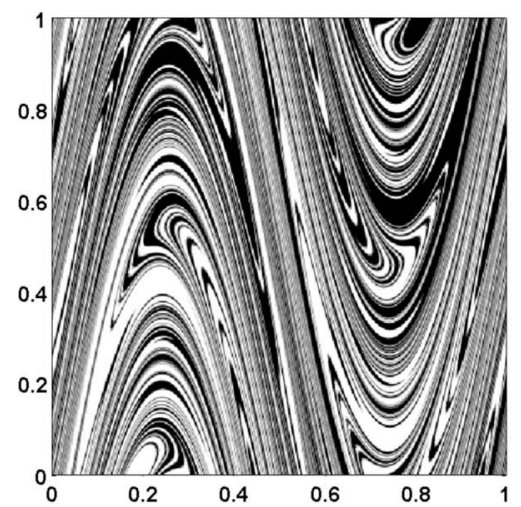

(d)

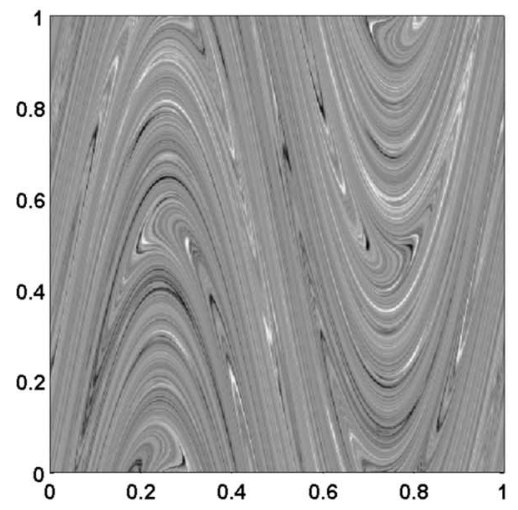

(g)

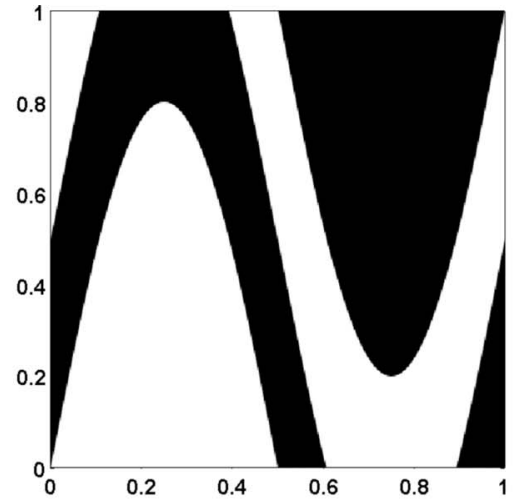

(b)

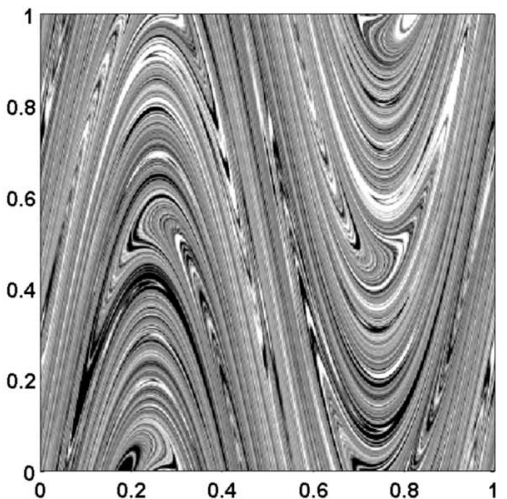

(e)

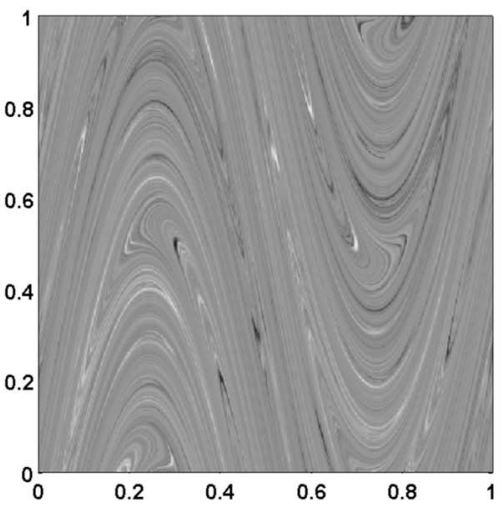

(h)

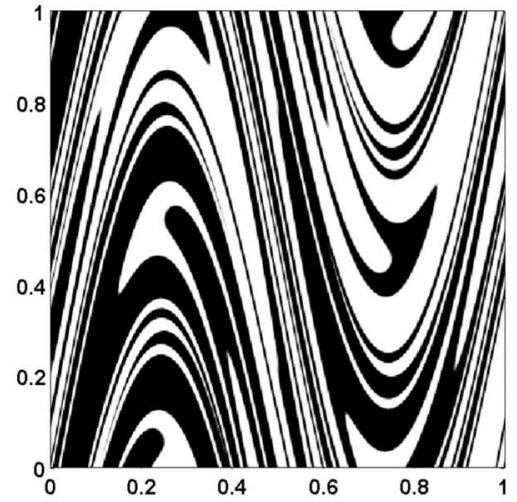

(c)

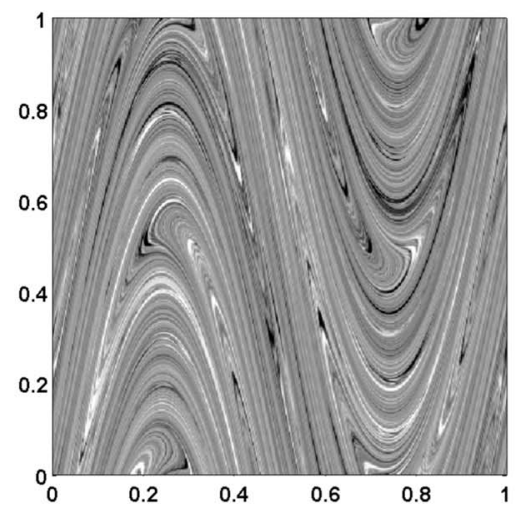

(f)

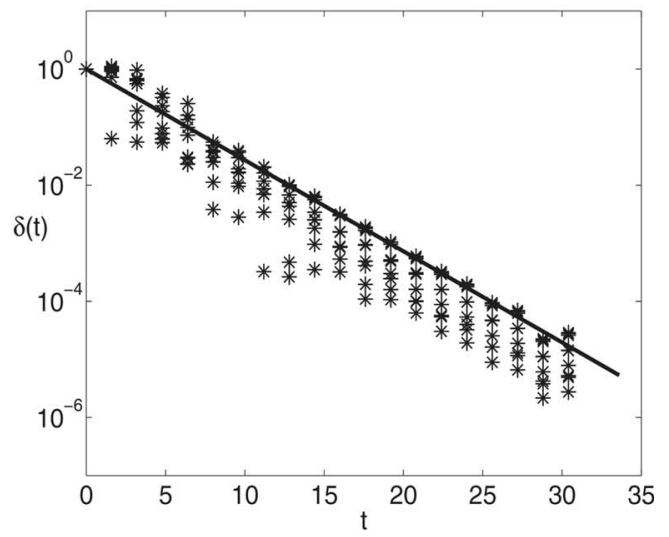

(i)

FIG. 6. Evolution of an initially inhomogeneous scalar distribution in the globally chaotic TPSF $(T=1.6)$ : (a) initial state $(C=1:$ white; $C=0$ : black); $[(b)-($ h) $]$ periodwise evolution of the scalar distribution; (i) decay of the local concentration in a sequence of positions on the line $y=3 / 4$ (markers) compared against the predicted homogenization time $\tau$ (sloping line).

$\lambda=1$ and $\lambda=-1$, respectively. The eigenvectors in panels (b)(d) clearly vanish outside the islands, meaning their active zones are evidently restricted to the latter. The eigenvector in panel (a) is constant yet nonzero outside the islands and is an example of a case where the eigenmode, due to its corresponding eigenvalue $\lambda=1$, must only be homogeneous outside its corresponding spatial entities (Sec. IV C). Hence, the eigenvectors associated with the islands are in agreement with Conjectures 3 and 5. The spatial structure of the eigenvectors reveals that the active zones of the eigenmodes with $\lambda=-1$ are restricted to the core of the islands, where they change sign every period, thus effectuating a persistent period-2 fluctuation around the fixed background distribution set up by the eigenmodes with $\lambda=1$ in the asymptotic advec- 

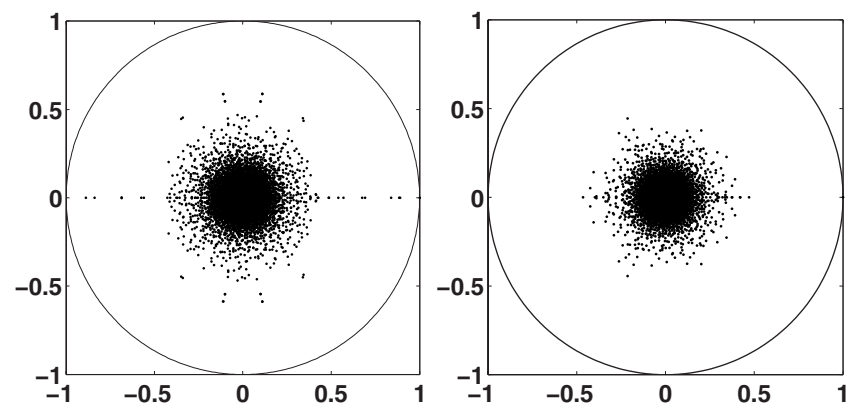

(a)

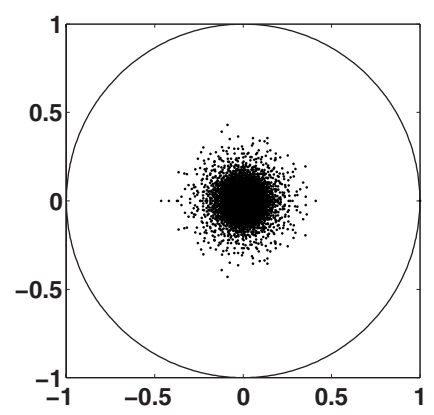

(c)

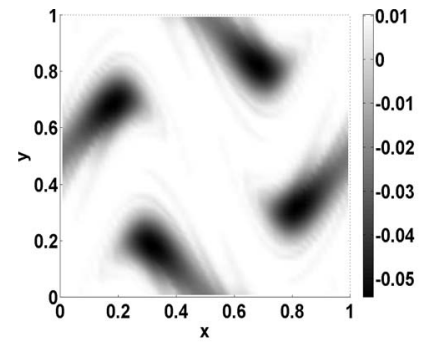

(a)

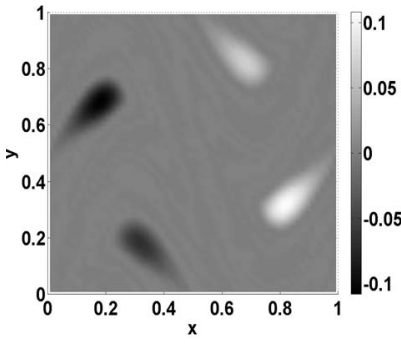

(c)

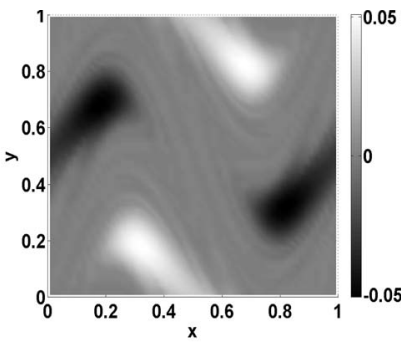

(b)

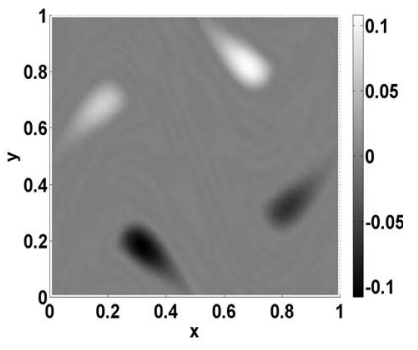

(d)

FIG. 9. Two period-2 pairs of persistent eigenmodes associated with the two period-2 island chains for the partially chaotic TPSF $(T=0.8)$ : [(a) and (b)] eigenvectors corresponding with $\lambda=1 ;[(\mathrm{c})$ and (d)] eigenvectors corresponding with $\lambda=-1$.

FIG. 7. Eigenvalue spectrum for the TPSF for a number of period times $T$ near the globally chaotic case $T=1.6$ : (a) $T=1.5$; (b) $T=1.7$; (c) $T=1.8$; (d) $T=1.9$.

tion pattern. The active zones of the latter modes include the fringes of the islands as well; here they establish a persistent heterogeneous scalar distribution.

The chaotic sea surrounding the islands is dominated by a period-4 cluster with eigenvalues $\Lambda=\lambda_{\text {dom }}\{1, i,-1,-i\}$, with $\lambda_{\text {dom }} \approx 0.84$, indicated by the small circles in Fig. 10(a). Here the dominant eigenvalue $\lambda=-\lambda_{\text {dom }}$, similar to that for $T=1.6$ (Fig. 5), also appears as multiple eigenvalues very close to one another. Numerical experiments again reveal that these eigenvalues (and associated eigenvectors) progress toward one physically meaningful state. The corresponding eigenvectors are shown in Figs. 10(b)-10(d), where panel (c) gives the absolute magnitude of the complex-conjugate pair

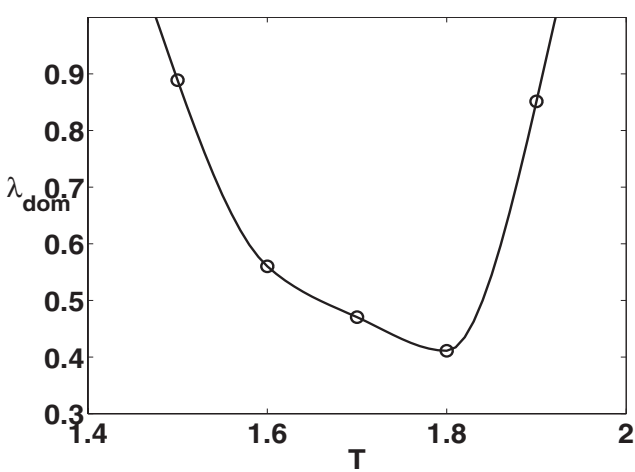

(a) of eigenvectors associated with the complex-conjugate pair of eigenvalues. The active zones of these eigenvectors combined cover the entire chaotic sea and vanish in the islands, in perfect agreement with Conjectures 3 and 5. The associated eigenmodes set up an exponential decay of heterogeneous scalar distributions toward a homogeneous state within the chaotic sea similar to that observed for the fully chaotic case $T=1$.6.

The central role of the periodic eigenmodes belonging to the above eigenvectors in the spatiotemporal evolution of scalar distributions is demonstrated in Fig. 11 for the same initial conditions as considered before for $T=1.6$. Shown is the state after every fourth period. The progression clearly reveals the persistent nature of the scalar distribution within the islands and its self-similar decay within the chaotic sea

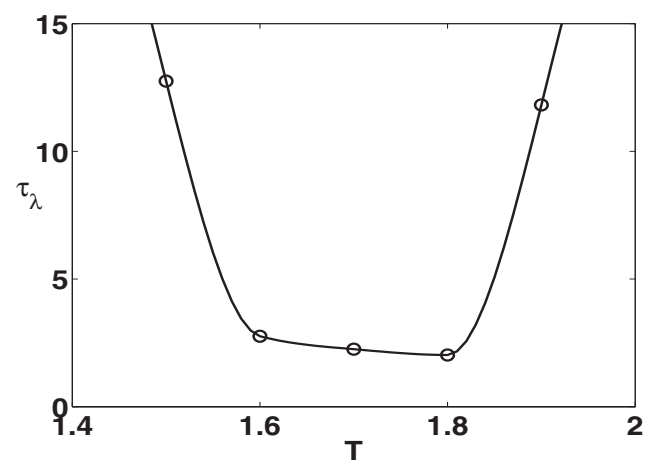

(b)

FIG. 8. Optimization of the mixing efficiency in the globally chaotic regime of the TPSF: (a) dominant eigenvalue $\lambda_{\text {dom }}$ vs $T$; (b) characteristic homogenization time $\tau_{\lambda}$ vs $T$. 


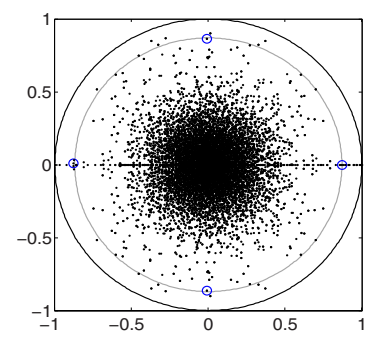

(a)

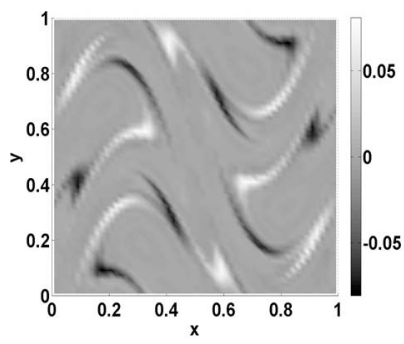

(c)

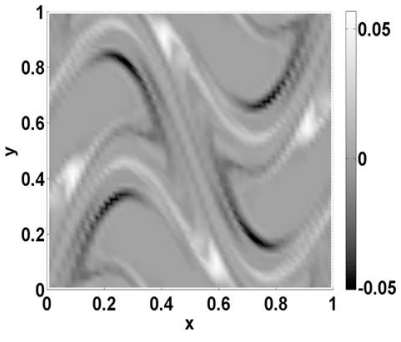

(b)

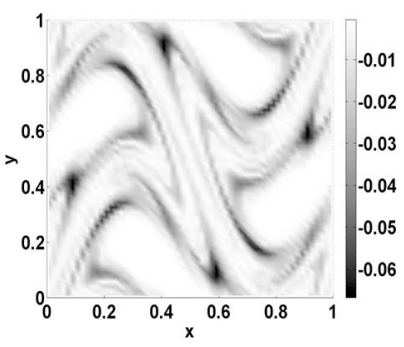

(d)
FIG. 10. (Color online) Period-4 cluster of decaying eigenmodes associated with the chaotic sea for the partially chaotic TPSF $(T=0.8)$ : (a) eigenvalue spectrum (period-4 cluster: small circles); [(b) and (c)] real eigenvectors; (d) magnitude of complex-conjugate eigenvectors.

during the four-period intervals, dictated by the above eigenmodes, that sets in after an initial nonperiodic stage. Note that in the actual evolution material is exchanged between the cores of diametrically opposite islands after each period by the fluctuating period- 2 modes. Furthermore, note the persistent heterogeneities at the fringes of the islands coinciding with the spatial structure of the persistent eigenmodes in Figs. 9(a) and 9(b). Figure 11(i) gives the decay of the local concentration $\delta(t)$, following Eq. (22), in a series of positions inside the chaotic sea (markers) compared against the predicted homogenization time $\tau_{\lambda}=-T / \ln \lambda_{\text {dom }}=4.68$ (sloping line). The simulated decay rates are again in excellent agreement with the prediction, although the oscillations about the predicted trend are substantially larger than in the fully chaotic case $T=1.6$ [Fig. 6(i)]. This further substantiates the assertion set forth in Sec. IV B that the eigenvalue spectrum provides both qualitative and quantitative insights into the transport properties of a given flow. However, it must be stressed that situations may be more complex than encountered here in that chaotic seas may comprise subregions with each their own dominant eigenmode. In such cases, regionwise homogenization times $\tau_{\lambda}$ can be assigned in basically the same manner as employed here on the basis of the local dominant eigenmodes.

The eigenvalues in between the unit circle and the circle delineated by the dominant eigenmodes [Fig. 10(a)] correspond to decaying eigenmodes active within the island regions. (The higher magnitude of the eigenvalues - and thus slower decay rates - than that of the dominant period-4 cluster implies restriction of these eigenmodes to the islands.) Two kinds can be distinguished. First are slowest-decaying eigenmodes associated with the core of the elliptic islands, determining the transient evolution toward the persistent period-2 fluctuation. (These modes thus act as dominant eigenmodes of the island cores.) Second are slowestdecaying eigenmodes associated with the fringes of the island regions, occupied by chaotic bands accommodating island chains. These regions emanate from disintegration of outer orbits of the elliptic islands and form the transition between the regular transports inside the island cores to the chaotic transport inside the chaotic sea. The formation of such regions is according to the Poincaré-Birkhoff theorem. ${ }^{28}$ This theorem states that orbits on which material elements perform rational motion (i.e., return to their initial position after a finite number of revolutions) disintegrate into chains of periodic points that are alternately elliptic and hyperbolic. This, in turn, manifests itself in the formation of an island chain and surrounding chaotic sea. [This transition is, e.g., also reflected in the "interlocking" of the outer nonhomogeneous features of the persistent eigenmode in Fig. 9(a) with the nonzero regions of the dominant eigenmode in Fig. 10(c).] The smallness of the spatial features inside the transitional regions here prohibits its full resolution, leading to (partial) "smearing out" of island chains and chaotic bands in the mapping representation. This manifests itself in said decaying eigenmodes that effectively represent the "average" dynamics in the fringes of the islands occupied by the beforementioned chaotic bands and island chains.

The case $T=0.56$, contrary to the above case $T=0.8$, exhibits island chains encircling period-1 islands that are of sufficient size to admit resolution by the mapping approach [Fig. 3(b)]. They form two period-6 chains that, in accordance with Conjecture 4, result in two period-6 clusters of eigenvalues $\Lambda=\left\{1, \omega, \omega^{2}, \omega^{3}, \omega^{4}, \omega^{5}\right\}$, with $\omega=e^{\pi i / 3}$, in the eigenvalue spectrum, indicated by the circles in Fig. 12(a) [compare to Fig. 2(f)]. One of the corresponding eigenvectors is given in Fig. 12(b), clearly demarcating the island chains.

A further quantitative mixing measure, besides the characteristic homogenization time $\tau_{\lambda}$, provided by the spectral decomposition is the relative nonmixing area (i.e., the combined area covered by all elliptic islands relative to the total area). The cellular approach of the mapping method facilitates straightforward computation of this measure via

$$
A_{N M}=\frac{\sum_{i=1}^{N_{1}} A_{i}}{\sum_{i=1}^{N} A_{i}},
$$

where $N_{1}$ is the number of cells containing the active region of eigenvectors with eigenvalues on the unit circle, $N$ is the total number of cells, and $A_{i}$ is the area of the $i$ th cell. [For uniform cell sizes, measure (23) simplifies to $A_{N M}=N_{1} / N$.] For example, in case $T=1.18$, the nonmixing zones correspond to the two period-2 island chains that, according to Eq. (23), occupy $5.96 \%$ of the total area.

\section{Some numerical issues}

The spectral decomposition and its analysis present a number of numerical challenges. The most important of those are addressed below.

The present analysis concerns distributive mixing purely by advection. However, the mapping method describes the 


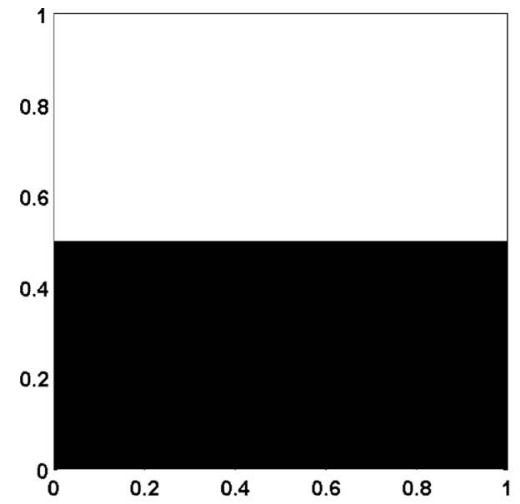

(a)

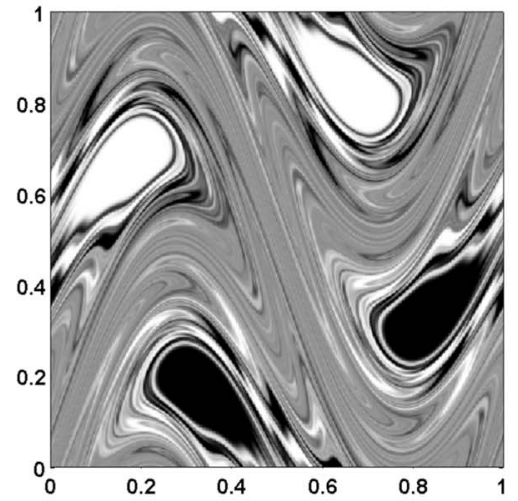

(d)

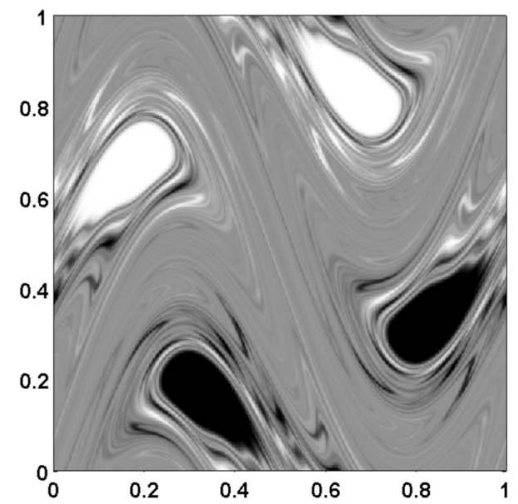

$(\mathrm{g})$

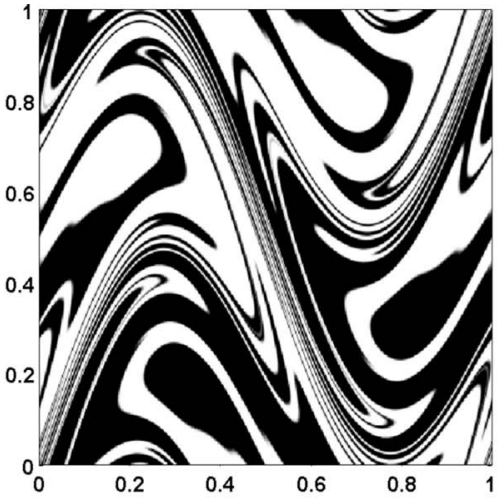

(b)

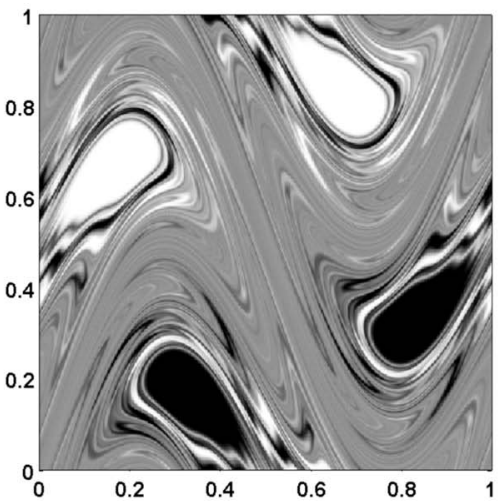

(e)

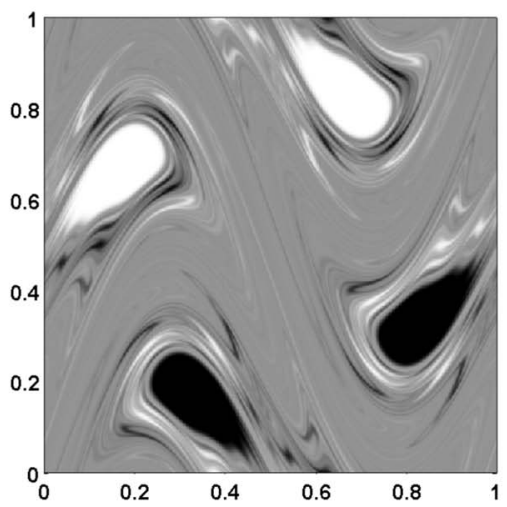

(h)

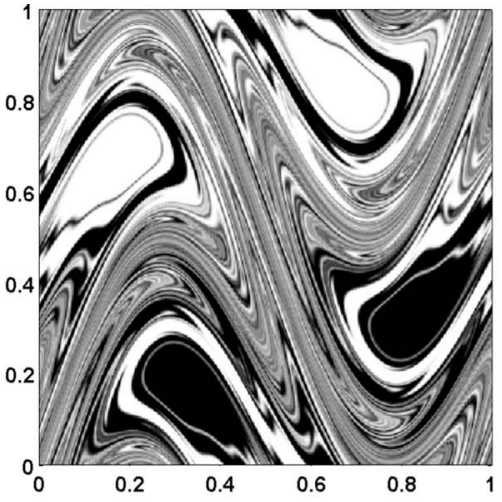

(c)

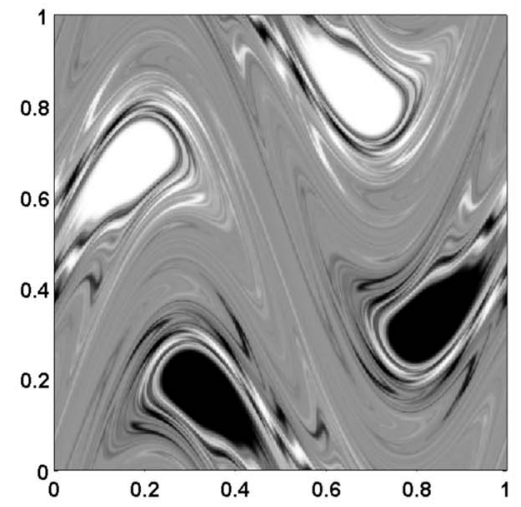

(f)

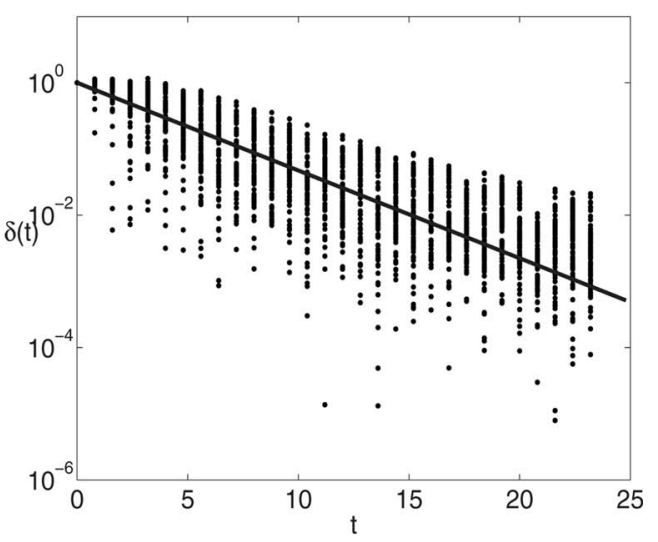

(i)

FIG. 11. Evolution of an initially inhomogeneous scalar distribution in the partially chaotic TPSF $(T=0.8)$ : (a) initial state $(C=1:$ white; $C=0$ : black); $[(b)-($ h) $]$ periodwise evolution of the scalar distribution; (i) decay of the local concentration in a sequence of positions inside the chaotic sea (markers) compared against the predicted homogenization time $\tau$ (sloping line).

transport properties through discrete approximation by cells of finite size and, consequently, suffers from numerical diffusion. ${ }^{23,24}$ Hence, the mapping method effectively represents advective-diffusive transport with very weak diffusion that diminishes with increasing spatial resolution yet remains nonzero for finite cells. The presence of diffusion, no matter how weak, implies that all eigenvalues of the mapping matrix must reside within the unit circle. Thus the computed eigenvalues associated with elliptic islands, although strictly located on the unit circle (Conjecture 1), always fall within this circle, exactly as observed in the above cases. Similarly, the eigenvalues corresponding with physically decaying eigenmodes will be slightly lower than their true counterparts, e.g., meaning that the above characteristic homogenization times are slightly overpredicted.

It is important to note in the context of chaotic mixing is 


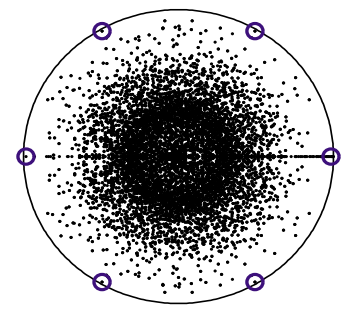

(a)

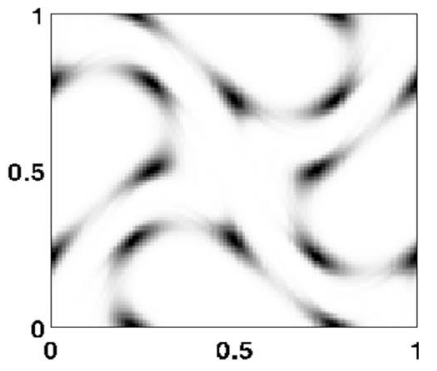

(b)
FIG. 12. (Color online) Two period-6 clusters of persistent eigenmodes associated with the two period- 6 island chains for the partially chaotic TPSF ( $T=0.56$ ): (a) eigenvalue spectrum (period-6 clusters: small circles); (b) magnitude of one of the corresponding eigenvectors; gray scale indicates level between zero (white) and maximum (black).

that the disintegration of islands (signifying nonmixing zones) into chaotic seas follows scenarios that repeat themselves ad infinitum within each island. ${ }^{28}$ Moreover, the manifolds in chaotic seas are infinitely long and intricately folded. This implies the formation of infinitesimally small spatial structures, which, irrespective of the resolution, a priori prohibits capturing every spatial detail with the mapping approach —or any other numerical method for that matter. Thus the mapping matrix always to some degree suffers from smearing out of spatial features (and thereby numerical diffusion) in case of (partially) chaotic systems. This manifests itself, e.g., in the formation of the decaying eigenmodes that represent the average dynamics in the fringes of the elliptic islands in the chaotic case $T=1.6$.

The fact that eigenvalues of the mapping matrix are always within the unit circle may significantly complicate numerical determination of the physical meaning of its corresponding eigenmodes in cases with only weak chaos and/or very small islands. Weak chaos implies slow decay rates and, inherently, eigenvalues of associated dominant eigenmodes relatively close to the unit circle; very small islands imply stronger numerical diffusion due to the difficulty of full numerical resolution and, inherently, eigenvalues relatively far inside the unit circle. Both cases result in very similar eigenvalue spectra in which differentiation between eigenmodes associated with islands and chaotic seas becomes very difficult if not impossible. This is further aggravated by the fact that finite resolution may lead to multiple eigenvectoreigenvalue pairs that are very close and, in fact, form approximations to one and the same physical eigenmode. [This, e.g., occurs for the dominant eigenvalues on the left part of the real axis in Figs. 5(a) and 10(a).] These effects render the eigenmode analysis of, in particular, partially chaotic cases as, e.g., the TPSF for $T=0.8$ very challenging.

\section{EIGENMODE ANALYSIS OF A REALISTIC THREE-DIMENSIONAL CASE: THE PARTITIONED-PIPE MIXER}

\section{A. Introduction}

The partitioned-pipe mixer (PPM) was introduced by Khakhar $e t a l .{ }^{36}$ as a simplification of the well-known Kenics static mixer and was representative of a wide range of indus-

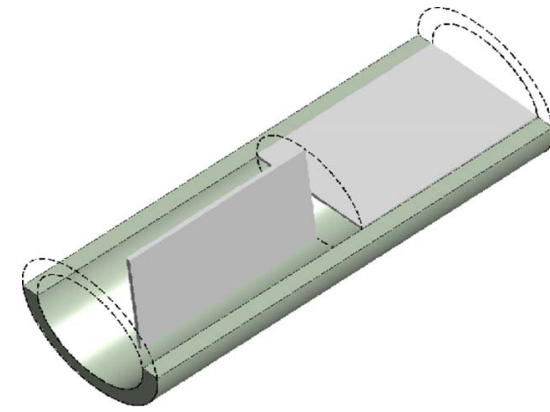

(a)

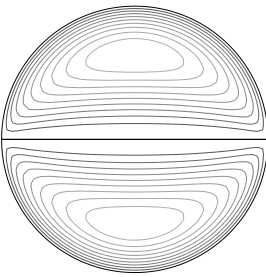

(b)
FIG. 13. (Color online) Schematic of the PPM: (a) perspective view (flow direction from right to left); (b) streamline portrait of the transverse flows on both sides of the plates.

trial mixing devices. The PPM consists of a cylindrical pipe of radius $R$ partitioned into a sequence of semicircular ducts by means of rectangular plates of length $L$ fixed perpendicularly to each other [Fig. 13(a)]. An axial throughflow is set up by imposing an axial pressure gradient and the plates induce a partitionwise transverse circulation according to Fig. 13(b). The arrangement of the plates effectuates systematic reorientation of the transverse flow and thus provides the necessary (yet by no means sufficient) conditions for chaotic mixing of the axial throughflow. ${ }^{22}$ The flow field within each partition is described by the analytical solution of Meleshko et al. ${ }^{37}$ The systematic reorientation of the plates means that the total flow field is spatially periodic and of form (5) with period length $Z=2 L$. The fluid dynamics and transport properties are governed by the single control parameter

$$
\beta=\frac{4 V_{\theta} L}{3 \kappa V_{Z} R},
$$

with $R$ and $L$ as before, $V_{\theta}$ as the azimuthal velocity at the cylinder wall, $V_{Z}$ as the mean axial flow, and $\kappa \approx 0.915$ as a constant. ${ }^{29,36}$

The spatial periodicity of the flow field in the PPM admits reduction in the three-dimensional flow to a twodimensional mapping between the inlets $z=2 k L$ of consecutive periodic units, with $k=0,1, \ldots$ (Refs. 29 and 36). This mapping is the spatial equivalent to the mapping between consecutive time levels $t=2 k T$ in two-dimensional timeperiodic flows as, e.g., the TPSF (Sec. V). Both Poincaré sections and the mapping matrix $\boldsymbol{\Phi}$ are based on this mapping between inlets. This equivalence between spatial and temporal mappings enables an eigenmode analysis of the mixing properties of the PPM similar to that performed for the TPSF in Sec. V.

\section{B. Eigenmode analysis}

The PPM always has nonmixing zones (i.e., elliptic islands) and, consequently, never accomplishes global chaos, or equivalently, efficient mixing. ${ }^{29}$ Presence, size, and location of such nonmixing zones are key determinants regarding the mixing ability of a given flow. Their ubiquity in the PPM lends this configuration well for studies on the existence and characteristics of nonmixing zones under realistic three- 


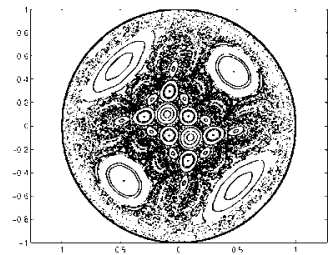

(a)

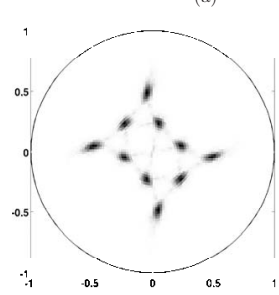

(c)

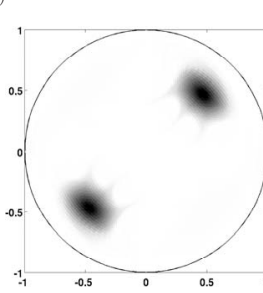

(f)

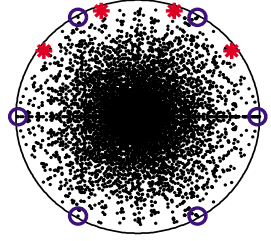

(b)

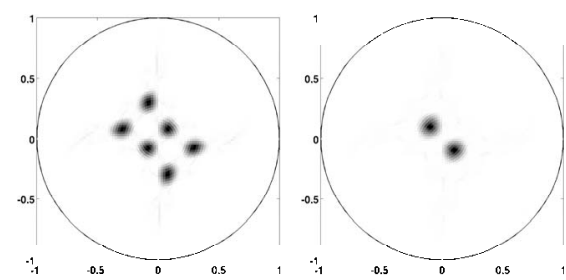

(e)

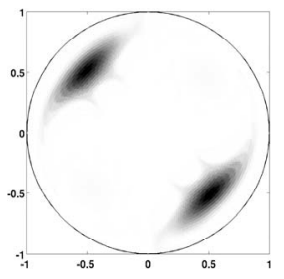

(g)

FIG. 14. (Color online) Periodic clusters of persistent eigenmodes corresponding with the nonmixing zones in the PPM at $\beta=1$ : (a) Poincaré section of the two-dimensional mapping of the PPM; (b) eigenvalue spectrum (period-6 cluster: circles; period-10 cluster: stars, only partially highlighted); (c) one of the eigenvectors of the period-10 chain; (d) one of the eigenvectors of the period-6 chain; (e) one of the eigenvectors of the period-2 pair; [(f) and (g)] two eigenvectors of the period-1 cluster. Shown is the eigenvector magnitude; gray scale indicates level between zero (white) and maximum (black).

dimensional conditions. The following analysis examines the connection between these nonmixing zones and the spectral decomposition of the mapping matrix for the PPM so as to further investigate (and demonstrate) the potential of the present eigenmode analysis for realistic mixing flows.

Figure 14(a) gives the Poincaré section of the PPM for $\beta=1$, which exposes an arrangement of islands of different periodicities with substantially greater complexity than the TPSF considered before: one period-10 island chain, one period- 6 island chain, one period- 2 island chain, and four isolated period-1 islands. The isolated period-10, period-6, and period-2 island chains correspond to clusters of eigenvalues on the unit circle according to Conjecture 4 for $p$ $=10, p=6$, and $p=2$, respectively, as indicated in the eigenvalue spectrum in Fig. 14(b). (For clarity only four of the eigenvalues of period-10 cluster are highlighted.) Note that period-2 eigenvalues coincide with the eigenvalues of period-6 cluster on the real axis. Figures 14(c)-14(e) give one of the associated eigenvectors for period-10, period-6, and period-2 clusters, respectively, the active regions (shaded) of which fully demarcate the corresponding island chains in the Poincaré section [Fig. 14(a)], consistent with Conjecture 5. Essentially equivalent to the case $T=0.8$ of the TPSF, each of the eigenvectors of a cluster thus fully exposes its related island chain; differences between eigenvectors ex- ist only in relative magnitude and sign within the islands in a similar manner, as shown in Fig. 9 for the TPSF at $T=0.8$.

The four eigenvectors associated with the four period-1 islands behave in a markedly different way in that they appear to "amalgamate" into two pairs that each demarcates only two of the four islands. Figures 14(f) and 14(g) show one eigenvector of each pair, with active regions again shaded. This demonstrates the inherent unpredictability of the role of the individual eigenvectors in the demarcation of the corresponding island group. In all previous cases of both TPSF and PPM each eigenvector belonging to an island group demarcates the entire group; here the eigenvectors only partially demarcate the group. The combined eigenvectors nonetheless fully cover this group in accordance with Conjecture 5 .

The case $\beta=4$ exhibits yet another form of individual contribution of eigenvectors of a period $p$ group to its total active region. The corresponding Poincaré section [Fig. 15(a)] reveals one isolated period-4 island chain and two period-2 island chains. Their eigenvalues cluster again on the unit circle following Eq. (18). The eigenvectors of period-4 chain behave as those of the isolated chains for case $\beta=1$ : The active region of each eigenvector, of which one is shown in Fig. 15(c), demarcates the entire period-4 island chain. The active regions of the four eigenvectors corresponding to the two period- 2 chains, on the other hand, pairwise demarcate either the one or the other period-2 chain. Figures 15(d) and 14(e) show one member of each pair, the active regions (shaded) of which coincide with one of the two period-2 island chains in Fig. 15(a). Thus here, strict separation between the eigenvectors occurs in that they belong to one of the chains only. However, the combined eigenvectors again fully demarcate the island group following Conjecture 5.

The above investigation revealed that the generic connection between the spectral decomposition of the mapping matrix $\boldsymbol{\Phi}$ and the advection properties, condensed in Conjectures 1-5 and examined before for the idealized TPSF, holds for the realistic PPM as well. The existence and characteristics of nonmixing zones, a key determinant in the mixing ability of a flow, follow readily from the spectral properties of $\boldsymbol{\Phi}$. This demonstrates the great potential of the eigenmode analysis of the mapping matrix for realistic threedimensional mixing flows.

\section{CONCLUSIONS}

The spectral (or eigenvector-eigenvalue) decomposition of the distribution matrix $\boldsymbol{\Phi}$ of a periodic flow obtained via the mapping method can be used to diagnose various kinematical aspects of distributive mixing. This spectral decomposition constitutes a discrete approximation to the eigenmodes of the continuous advection operator (or Floquet operator) in time-periodic and spatially periodic flows. Mass conservation always results in one trivial (i.e., uniform) eigenvector corresponding to eigenvalue $\lambda=1$, irrespective of the mixing properties; the transport properties are determined by the remaining nontrivial eigenvector-eigenvalue pairs. Reconciliation of the mixing characteristics with these eigenvector-eigenvalue pairs on the basis of available knowl- 


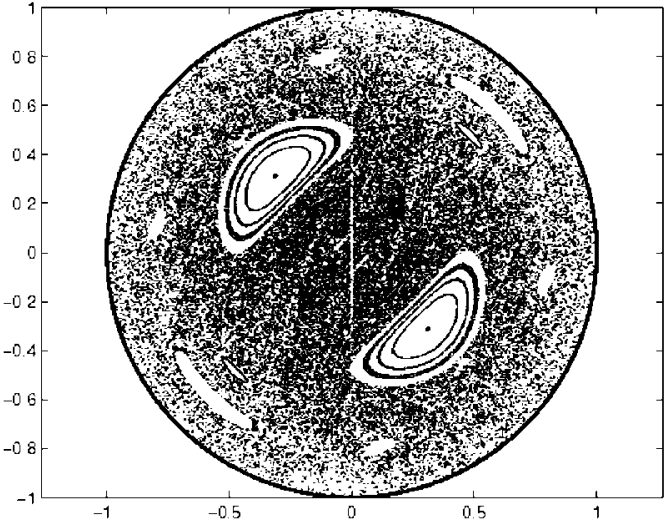

(a)

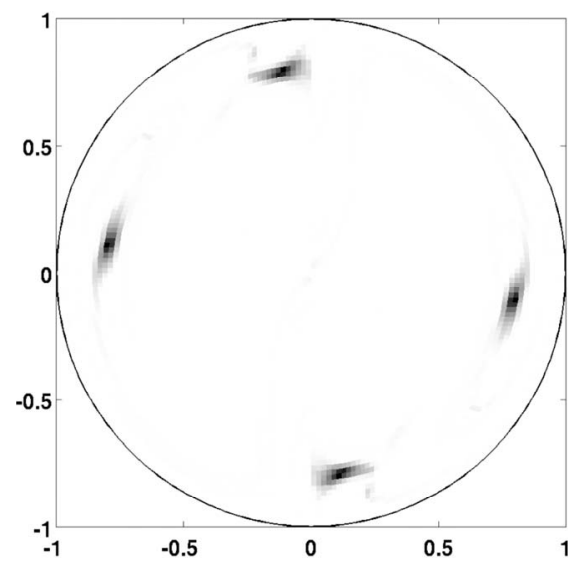

(c)

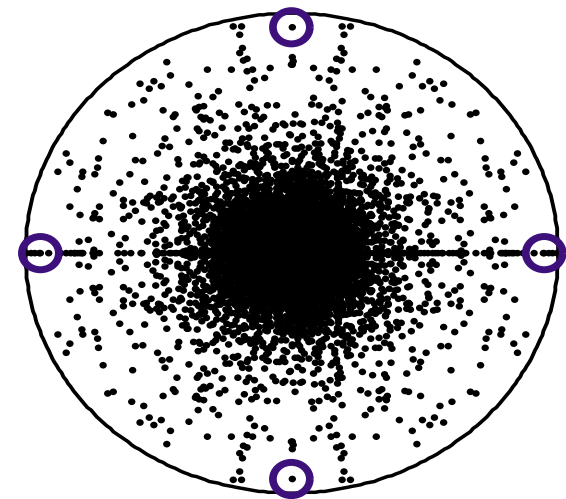

(b)

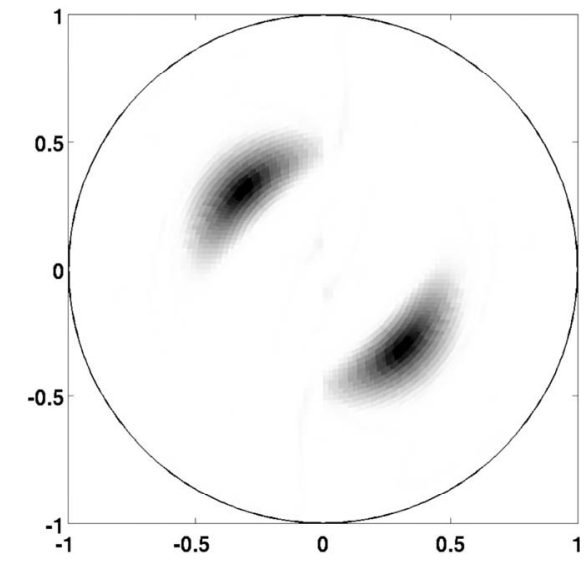

(d)

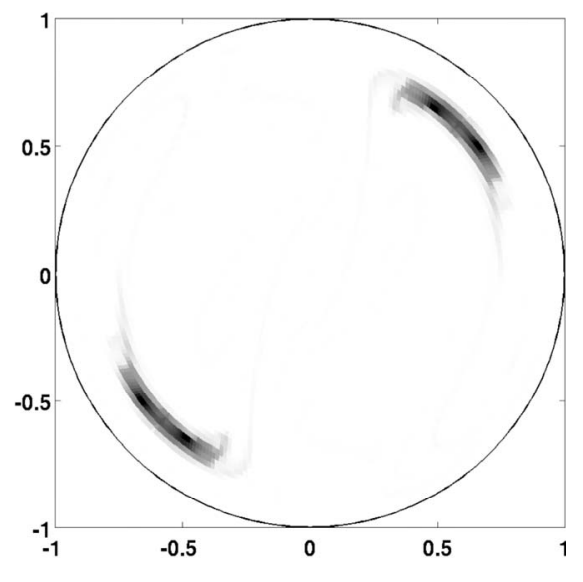

(e)

FIG. 15. (Color online) Periodic clusters of persistent eigenmodes corresponding with the nonmixing zones in the PPM at $\beta=4$ : (a) Poincaré section of the two-dimensional mapping of the PPM; (b) eigenvalue spectrum (period-2/4 clusters: circles); (c) one of the eigenvectors of the period-4 chain; [(d) and (e)] two eigenvectors of the period-2 cluster. Shown is the eigenvector magnitude; gray scale indicates level between zero (white) and maximum (black).

edge on mixing flows is the key objective of the study.

The distribution of the nontrivial eigenvalues in the complex plane provides first insight into the mixing characteristics of the flow: Absence of eigenvalues on the unit circle and a dense concentration around the origin are signatures of chaotic mixing; presence of eigenvalues on the unit circle signifies nonmixing zones (i.e., elliptic islands and thus is a first qualitative indicator of inefficient mixing). The asymptotic advection pattern is governed by periodic eigenmodes [i.e., with argument of the eigenvalues commensurate with $2 \pi: \arg (\lambda)=2 \pi n / p$ ] that correspond to periodic points and their associated spatial entities (elliptic islands and chaotic seas). These eigenmodes emerge in clusters, consistent with the clustering of periodic points, according to their periodicity; e.g., a period- 4 island chain results in four eigenvalues $\lambda=e^{2 \pi i n / p}$, with $0 \leq n \leq p-1$ and $p=4$, equally spaced on the unit circle and four corresponding eigenvectors. The eigenvectors combined demarcate the associated island chain(s) or chaotic sea(s) and the activity of its corresponding eigenmodes is restricted to these spatial entities.

The asymptotic state of fully chaotic mixing is dominated by (clusters of) periodic eigenmode(s) corresponding with (clusters of) eigenvalue(s) of equal magnitude closest to the unit circle [dominant eigenmode(s)]. This magnitude determines the decay rate, or equivalently, the characteristic homogenization time; the corresponding eigenvector(s) determine(s) the asymptotic mixing pattern. The closer the magnitude is to the origin, the faster is the homogenization by the chaotic mixing. Hence, this magnitude can be used as a quantitative mixing measure for comparison of different mixing protocols.

The eigenmode analysis is demonstrated for two different mixing flows: the TPSF and the spatially periodic PPM. Investigation of fully chaotic and partially chaotic cases of the TPSF confirmed the conjectured relation between the spectral decomposition of $\boldsymbol{\Phi}$ and the transport properties, as summarized above. The (asymptotic) evolution of the scalar distribution in the fully chaotic TPSF is indeed governed by dominant periodic eigenmodes that correspond to a cluster of dominant eigenvalues of $\boldsymbol{\Phi}$. Moreover, the decay rate proves in excellent agreement with the characteristic homogenization time predicted on the basis of the magnitude of these eigenvalues. This homogenization time is easily computed and thus well suited for optimization of the mixing effi- 
ciency. For partially chaotic cases, periodic eigenmodes associated with the islands and the chaotic sea have been identified, corresponding to clusters of eigenvalues on the unit circle and within this circle, respectively. The active regions of the corresponding eigenvectors demarcate the islands and the chaotic sea. The area covered by these regions (i.e., the total nonmixing area) can be readily computed and provides, besides the homogenization time, a further quantitative mixing measure. The evolution within the chaotic sea is dominated by its associated eigenmodes in the same way as in the fully chaotic case. However, identification of dominant eigenmodes of the chaotic sea, in contrast with the eigenmodes of the islands, is difficult due to the presence of decaying eigenmodes relating to the transient evolution within the islands as well as their disintegrating outer orbits that have eigenvalues with magnitude comparable to those of the chaotic sea.

The findings on the idealized TPSF are substantiated by investigation of the realistic PPM flow. Nonmixing zones in the PPM are, as for the TPSF, also unambiguously tied to clusters of eigenvalues on the unit circle and demarcated by their corresponding eigenvectors. This link facilitates efficient and robust identification of nonmixing zones from the spectral decomposition of the corresponding mapping matrix. Similarly, isolation of the dominant eigenmodes of chaotic seas, demonstrated for the TPSF, provides qualitative and quantitative insights into the mixing characteristics. The present study thus demonstrates the potential of the eigenmode analysis for the investigation of the mixing characteristics of realistic periodic flows.

\section{ACKNOWLEDGMENTS}

The authors are grateful to the Dutch Polymer Institute (DPI) for financial support (Grant No. 446). P.D.A. thanks Professor Bud Homsy from UCSB for the fruitful discussions on this subject.

${ }^{1}$ R. T. Pierrehumbert, "Tracer microstructure in the large-eddy dominated regime," Chaos, Solitons Fractals 4, 774 (1994).

${ }^{2}$ W. Liu and G. Haller, "Strange eigenmodes and decay of variance in the mixing of diffusive tracers," Physica D 188, 1 (2004).

${ }^{3}$ J. Vanneste, "Intermittency of passive-scalar decay: Strange eigenmodes in random shear flows," Phys. Fluids 18, 087108 (2006).

${ }^{4}$ O. V. Popovych, A. Pikovsky, and B. Eckhardt, "Abnormal mixing of passive scalars in chaotic flows," Phys. Rev. E 75, 036308 (2007).

${ }^{5}$ A. Adrover, S. Cerbelli, and M. Giona, "On the interplay between advection and diffusion in closed laminar chaotic flows," J. Phys. Chem. A 105, 4908 (2001).

${ }^{6}$ A. Adrover, S. Cerbelli, and M. Giona, "A spectral approach to reaction/ diffusion kinetics in chaotic flows," Comput. Chem. Eng. 26, 125 (2002).

${ }^{7}$ S. Cerbelli, A. Adrover, and M. Giona, "Enhanced diffusion regimes in bounded chaotic flows," Phys. Lett. A 312, 355 (2003).

${ }^{8}$ S. Cerbelli, V. Vitacolonna, A. Adrover, and M. Giona, "Eigenvalueeigenfunction analysis of infinitely fast reactions and micromixing regimes in regular and chaotic bounded flows," Chem. Eng. Sci. 59, 2125 (2004).

${ }^{9}$ S. Cerbelli, A. Adrover, F. Creta, and M. Giona, "Foundations of laminar chaotic mixing and spectral theory of linear operators," Chem. Eng. Sci. 61, 2754 (2006).

${ }^{10}$ D. R. Lester, M. Rudman, G. Metcalfe, and H. M. Blackburn, "Global parametric solutions of scalar transport," J. Comput. Phys. 227, 3032 (2008).

${ }^{11}$ D. Rothstein, E. Henry, and J. P. Gollub, "Persistent patterns in transient chaotic fluid mixing," Nature (London) 401, 770 (1999).

${ }^{12}$ G. A. Voth, T. C. Saint, G. Dobler, and J. P. Gollub, "Mixing rates and symmetry breaking in two-dimensional chaotic flow," Phys. Fluids 15, 2560 (2003).

${ }^{13}$ R. Spencer and R. Wiley, "The mixing of very viscous liquids," J. Colloid Sci. 6, 133 (1951).

${ }^{14}$ P. G. M. Kruijt, O. S. Galaktionov, P. D. Anderson, G. W. M. Peters, and H. E. H. Meijer, "Analyzing mixing in periodic flows by distribution matrices: Mapping method," AIChE J. 47, 1005 (2001).

${ }^{15}$ O. S. Galaktionov, P. D. Anderson, G. W. M. Peters, and H. E. H. Meijer, "Analysis and optimization of Kenics mixers," Int. Polym. Process. 18, 138 (2003).

${ }^{16}$ O. S. Galaktionov, P. D. Anderson, G. W. M. Peters, and H. E. H. Meijer, "Mapping approach for 3D laminar mixing simulation: Application to industrial flows," Int. J. Numer. Methods Fluids 40, 345 (2002).

${ }^{17}$ M. K. Singh, P. D. Anderson, M. F. M. Speetjens, and H. E. H. Meijer, "Optimizing the rotated arc mixer," AIChE J. 54, 2809 (2008).

${ }^{18}$ P. D. Anderson, O. S. Galaktionov, G. W. M. Peters, H. E. H. Meijer, and C. L. Tucker, "Material stretching in laminar mixing flows: Extended mapping technique applied to the journal bearing flow," Int. J. Numer. Methods Fluids 40, 189 (2002).

${ }^{19}$ M. Giona, V. Vitacolonna, S. Cerbelli, and A. Adrover, "Advection diffusion in nonchaotic flows: Non-Hermitian operators, universality, and localization," Phys. Rev. E 70, 046224 (2004).

${ }^{20}$ G. I. Barenblatt, Scaling, Self-similarity, and Intermediate Asymptotics (Cambridge University Press, Cambridge, 1996).

${ }^{21}$ G. Haller, "Distinguished material surfaces and coherent structures in three-dimensional fluid flows," Physica D 149, 248 (2001).

${ }^{22}$ M. Speetjens, G. Metcalfe, and M. Rudman, "Topological mixing study of non-Newtonian duct flows," Phys. Fluids 18, 103103 (2006).

${ }^{23}$ M. K. Singh, T. G. Kang, H. E. H. Meijer, and P. D. Anderson, "The mapping method as a toolbox to analyze, design and optimize micromixers," Microfluid. Nanofluid. 5, 313 (2008).

${ }^{24}$ T. G. Kang, M. K. Singh, T. H. Kwon, and P. D. Anderson, "Chaotic mixing using periodic and aperiodic sequences of mixing protocols in a micromixer," Microfluid. Nanofluid. 4, 589 (2008).

${ }^{25}$ M. K. Singh, O. S. Galaktionov, H. E. H. Meijer, and P. D. Anderson, “A simplified approach to compute distribution matrices for the mapping method," Comput. Chem. Eng. 33, 1354 (2009).

${ }^{26} \mathrm{H}$. Aref, "The development of chaotic advection," Phys. Fluids 14, 1315 (2002).

${ }^{27}$ V. V. Meleshko and G. W. M. Peters, "Periodic points for two-dimensional Stokes flow in a rectangular cavity," Phys. Lett. A 216, 87 (1996).

${ }^{28}$ E. Ott, Chaos in Dynamical Systems (Cambridge University Press, Cambridge, 2002).

${ }^{29}$ J. M. Ottino, The Kinematics of Mixing: Stretching, Chaos, and Transport (Cambridge University Press, Cambridge, 1989).

${ }^{30}$ J. M. Ottino, "Mixing, chaotic advection and turbulence," Annu. Rev. Fluid Mech. 22, 207 (1990).

${ }^{31}$ G. Strang, Linear Algebra and its Applications (Harcourt Brace Jovanovich, San Diego, 1976).

${ }^{32}$ E. Kreyszig, Advanced Engineering Mathematics (Wiley, Chichester, 1999).

${ }^{33}$ M. F. M. Speetjens, H. J. H. Clercx, and G. J. F. van Heijst, "Inertiainduced coherent structures in a time-periodic viscous mixing flow," Phys. Fluids 18, 083603 (2006).

${ }^{34}$ J. G. Franjione and J. M. Ottino, "Symmetry concepts for the geometric analysis of mixing flows," Philos. Trans. R. Soc. London, Ser. A 338, 301 (1992).

${ }^{35}$ M. Liu, F. J. Muzzio, and R. L. Peskin, "Quantification of mixing in aperiodic chaotic flows," Chaos, Solitons Fractals 4, 869 (1994).

${ }^{36}$ D. V. Khakhar, J. G. Franjione, and J. M. Ottino, "A case study of chaotic mixing in deterministic flows: The partitioned pipe mixer," Chem. Eng. Sci. 42, 2909 (1987).

${ }^{37}$ V. V. Meleshko, O. S. Galaktionov, G. W. M. Peters, and H. E. H. Meijer, "Three-dimensional mixing in Stokes flow: The partitioned pipe mixer problem revisited," Eur. J. Mech. B/Fluids 18, 783 (1999). 